\title{
A new mapping for finding a common element of the sets of fixed points of two finite families of nonexpansive and strictly pseudo-contractive mappings and two sets of variational inequalities in uniformly convex and 2-smooth Banach spaces
}

Atid Kangtunyakarn*

\section{"Correspondence:}

beawrock@hotmail.com

Department of Mathematics,

Faculty of Science, King Mongkut's

Institute of Technology Ladkrabang,

Bangkok 10520, Thailand

\begin{abstract}
In this paper we introduce a new mapping in a uniformly convex and 2-smooth Banach space to prove a strong convergence theorem for finding a common element of the set of fixed points of a finite family of nonexpansive mappings and the set of fixed points of a finite family of strictly pseudo-contractive mappings and two sets of solutions of variational inequality problems. Moreover, we also obtain a strong convergence theorem for a finite family of the set of solutions of variational inequality problems and the set of fixed points of a finite family of strictly pseudo-contractive mappings by using our main result.
\end{abstract}

Keywords: nonexpansive mapping; strictly pseudo-contractive mapping; variational inequality problem

\section{Introduction}

Throughout this paper, we use $E$ and $E^{*}$ to denote a real Banach space and a dual space of $E$, respectively. For any pair $x \in E$ and $f \in E^{*},\langle x, f\rangle$ instead of $f(x)$. The duality mapping $J: E \rightarrow 2^{E^{*}}$ is defined by $J(x)=\left\{x^{*} \in E^{*}:\left\langle x, x^{*}\right\rangle=\|x\|^{2},\|x\|=\left\|x^{*}\right\|\right\}$ for all $x \in E$. It is well known that if $E$ is a Hilbert space, then $J=I$, where $I$ is the identity mapping. Recall the following definitions.

Definition 1.1 A Banach space $E$ is said to be uniformly convex iff for any $\epsilon, 0<\epsilon \leq 2$, the inequalities $\|x\| \leq 1,\|y\| \leq 1$ and $\|x-y\| \geq \epsilon$ imply there exists a $\delta>0$ such that $\left\|\frac{x+y}{2}\right\| \leq$ $1-\delta$.

Definition 1.2 A Banach space $E$ is said to be smooth if for each $x \in S_{E}=\{x \in E:\|x\|=1\}$, there exists a unique functional $j_{x} \in E^{*}$ such that $\left\langle x, j_{x}\right\rangle=\|x\|$ and $\left\|j_{x}\right\|=1$.

It is obvious that if $E$ is smooth, then $J$ is single-valued which is denoted by $j$.

\section{Springer}

( 2013 Kangtunyakarn; licensee Springer. This is an Open Access article distributed under the terms of the Creative Commons Attribution License (http://creativecommons.org/licenses/by/2.0), which permits unrestricted use, distribution, and reproduction in any medium, provided the original work is properly cited. 
Definition 1.3 Let $E$ be a Banach space. Then a function $\rho_{E}: \mathbb{R}^{+} \rightarrow \mathbb{R}^{+}$is said to be the modulus of smoothness of $E$ if

$$
\rho_{E}(t)=\sup \left\{\frac{\|x+y\|+\|x-y\|}{2}-1:\|x\|=1,\|y\|=t\right\} .
$$

A Banach space $E$ is said to be uniformly smooth if

$$
\lim _{t \rightarrow 0} \frac{\rho_{E}(t)}{t}=0 .
$$

It is well known that every uniformly smooth Banach space is smooth.

Let $q>1$. A Banach space $E$ is said to be $q$-uniformly smooth if there exists a fixed constant $c>0$ such that $\rho_{E}(t) \leq c t^{q}$. It is easy to see that if $E$ is $q$-uniformly smooth, then $q \leq 2$ and $E$ is uniformly smooth.

A mapping $T: C \rightarrow C$ is called a nonexpansive mapping if

$$
\|T x-T y\| \leq\|x-y\|
$$

for all $x, y \in C$.

$T$ is called an $\eta$-strictly pseudo-contractive mapping if there exists a constant $\eta \in(0,1)$ such that

$$
\langle T x-T y, j(x-y)\rangle \leq\|x-y\|^{2}-\eta\|(I-T) x-(I-T) y\|^{2}
$$

for every $x, y \in C$ and for some $j(x-y) \in J(x-y)$. It is clear that (1.1) is equivalent to the following:

$$
\langle(I-T) x-(I-T) y, j(x-y)\rangle \geq \eta\|(I-T) x-(I-T) y\|^{2}
$$

for every $x, y \in C$ and for some $j(x-y) \in J(x-y)$. We give some examples for a strictly pseudo-contractive mapping as follows.

Example 1.1 Let $\mathbb{R}$ be a real line endowed with the Euclidean norm and let $C=(0, \infty)$. Define the mapping $T: C \rightarrow C$ by

$$
T x=\frac{2 x^{2}}{3+2 x}, \quad \forall x \in C
$$

Then $T$ is a $\frac{1}{9}$-strictly pseudo-contractive mapping.

Example 1.2 (See [1]) Let $\mathbb{R}$ be a real line endowed with the Euclidean norm. Let $C=$ $[-1,1]$ and let $T: C \rightarrow C$ be defined by

$$
T x= \begin{cases}x & \text { if } x \in[-1,0] ; \\ x-x^{2} & \text { if } x \in(0,1] .\end{cases}
$$

Then $T$ is a $\lambda$-strictly pseudo-contractive mapping where $\lambda \leq \min \left\{\lambda_{1}, \lambda_{2}\right\}$ and $\lambda_{1} \leq \frac{1}{2}$, $\lambda_{2}<1$. 
Let $C$ and $D$ be nonempty subsets of a Banach space $E$ such that $C$ is nonempty closed convex and $D \subset C$, then a mapping $P: C \rightarrow D$ is sunny [2] provided $P(x+t(x-P(x)))=P(x)$ for all $x \in C$ and $t \geq 0$, whenever $x+t(x-P(x)) \in C$. A mapping $P: C \rightarrow D$ is called a retraction if $P x=x$ for all $x \in D$. Furthermore, $P$ is a sunny nonexpansive retraction from $C$ onto $D$ if $P$ is a retraction from $C$ onto $D$ which is also sunny and nonexpansive.

Subset $D$ of $C$ is called a sunny nonexpansive retraction of $C$ if there exists a sunny nonexpansive retraction from $C$ onto $D$.

An operator $A$ of $C$ into $E$ is said to be accretive if there exists $j(x-y) \in J(x-y)$ such that

$$
\langle A x-A y, j(x-y)\rangle \geq 0, \quad \forall x, y \in C .
$$

A mapping $A: C \rightarrow E$ is said to be $\alpha$-inverse strongly accretive if there exist $j(x-y) \in$ $J(x-y)$ and $\alpha>0$ such that

$$
\langle A x-A y, j(x-y)| \geq \alpha\|A x-A y\|^{2}, \quad \forall x, y \in C .
$$

Remark 1.3 From (1.1) and (1.2), if $T$ is an $\eta$-strictly pseudo-contractive mapping, then $I-T$ is $\eta$-inverse strongly accretive.

The variational inequality problem in a Banach space is to find a point $x^{*} \in C$ such that for some $j\left(x-x^{*}\right) \in J\left(x-x^{*}\right)$,

$$
\left\langle A x^{*}, j\left(x-x^{*}\right)\right| \geq 0, \quad \forall x \in C .
$$

This problem was considered by Aoyama et al. [3]. The set of solutions of the variational inequality in a Banach space is denoted by $S(C, A)$, that is,

$$
S(C, A)=\{u \in C:\langle A u, J(v-u)| \geq 0, \forall v \in C\} .
$$

Several problems in pure and applied science, numerous problems in physics and economics reduce to finding an element in (1.4); see, for instance, [4-6].

Recall that normal Mann's iterative process was introduced by Mann [7] in 1953. The normal Mann's iterative process generates a sequence $\left\{x_{n}\right\}$ in the following manner:

$$
\left\{\begin{array}{l}
x_{1} \in C, \\
x_{n+1}=\left(1-\alpha_{n}\right) x_{n}+\alpha_{n} T x_{n}, \quad \forall n \geq 1,
\end{array}\right.
$$

where the sequence $\left\{\alpha_{n}\right\} \subset(0,1)$. If $T$ is a nonexpansive mapping with a fixed point and the control sequence $\left\{\alpha_{n}\right\}$ is chosen so that $\sum_{n=1}^{\infty} \alpha_{n}\left(1-\alpha_{n}\right)=\infty$, then the sequence $\left\{x_{n}\right\}$ generated by normal Mann's iterative process (1.5) converges weakly to a fixed point of $T$.

In 1967, Halpern has introduced the iteration method guaranteeing the strong convergence as follows:

$$
\left\{\begin{array}{l}
x_{1} \in C, \\
x_{n+1}=\left(1-\alpha_{n}\right) x_{1}+\alpha_{n} T x_{n}, \quad \forall n \geq 1,
\end{array}\right.
$$


where $\left\{\alpha_{n}\right\} \subset(0,1)$. Such an iteration is called Halpern iteration if $T$ is a nonexpansive mapping with a fixed point. He also pointed out that the conditions $\lim _{n \rightarrow \infty} \alpha_{n}=0$ and $\sum_{n=1}^{\infty} \alpha_{n}=\infty$ are necessary for the strong convergence of $\left\{x_{n}\right\}$ to a fixed point of $T$.

Many authors have modified the iteration (1.6) for a strong convergence theorem; see, for instance, [8-10].

In 2008, Zhou [11] proved a strong convergence theorem for the modification of normal Mann's iteration algorithm generated by a strict pseudo-contraction in a real 2-uniformly smooth Banach space as follows.

Theorem 1.4 Let $C$ be a closed convex subset of a real 2-uniformly smooth Banach space $E$ and let $T: C \rightarrow C$ be a $\lambda$-strict pseudo-contraction such that $F(T) \neq \emptyset$. Given $u, x_{0} \in C$ and sequences $\left\{\alpha_{n}\right\},\left\{\beta_{n}\right\},\left\{\gamma_{n}\right\}$ and $\left\{\delta_{n}\right\}$ in $(0,1)$, the following control conditions are satisfied:

(i) $a \leq \alpha_{n} \leq \frac{\lambda}{K^{2}}$ for some $a>0$ and for all $n \geq 0$,

(ii) $\beta_{n}+\gamma_{n}+\delta_{n}=1$ for all $n \geq 0$,

(iii) $\lim _{n \rightarrow \infty} \beta_{n}=0$ and $\sum_{n=1}^{\infty} \beta_{n}=\infty$,

(iv) $\alpha_{n+1}-\alpha_{n} \rightarrow 0$, as $n \rightarrow \infty$,

(v) $\quad 0<\liminf _{n \rightarrow \infty} \gamma_{n} \leq \limsup _{n \rightarrow \infty} \gamma_{n}<1$.

Let a sequence $\left\{x_{n}\right\}$ be generated by

$$
\left\{\begin{array}{l}
y_{n}=\alpha_{n} T x_{n}+\left(1-\alpha_{n}\right) x_{n}, \\
x_{n+1}=\beta_{n} u+\gamma_{n} x_{n}+\delta_{n} y_{n}, \quad n \geq 0 .
\end{array}\right.
$$

Then $\left\{x_{n}\right\}$ converges strongly to $x^{*} \in F(T)$, where $x^{*}=Q_{F(T)}(u)$ and $Q_{F(T)}: C \rightarrow F(T)$ is the unique sunny nonexpansive retraction from $C$ onto $F(T)$.

In 2006, Aoyama et al. introduced a Halpern-type iterative sequence and proved that such a sequence converges strongly to a common fixed point of nonexpansive mappings as follows.

Theorem 1.5 Let E be a uniformly convex Banach space whose norm is uniformly Gâteaux differentiable and let $C$ be a nonempty closed convex subset of $E$. Let $\left\{T_{n}\right\}$ be a sequence of nonexpansive mappings of $C$ into itself such that $\bigcap_{n=1}^{N} F\left(T_{i}\right)$ is nonempty and let $\left\{\alpha_{n}\right\}$ be a sequence of $[0,1]$ such that $\lim _{n \rightarrow \infty} \alpha_{n}=0$ and $\sum_{n=1}^{\infty} \alpha_{n}=\infty$. Let $\left\{x_{n}\right\}$ be a sequence of $C$ defined as follows: $x_{1}=x \in C$ and

$$
x_{n+1}=\alpha_{n} x+\left(1-\alpha_{n}\right) T_{n} x_{n}
$$

for every $n \in \mathbb{N}$. Suppose that $\sum_{n=1}^{\infty} \sup \left\{\left\|T_{n+1} z-T_{n} z\right\|: z \in B\right\}<\infty$ for any bounded subset $B$ of $C$. Let $T$ be a mapping of $C$ into itself defined by $T z=\lim _{n \rightarrow \infty} T_{n} z$ for all $z \in C$ and 
suppose that $F(T)=\bigcap_{n=1}^{\infty} F\left(T_{n}\right)$. If either

(i) $\sum_{n=1}^{\infty}\left|\alpha_{n+1}-\alpha_{n}\right|<\infty$ or

(ii) $\quad \alpha_{n} \in(0,1]$ for every $n \in \mathbb{N}$ and $\lim _{n \rightarrow \infty} \frac{\alpha_{n}}{\alpha_{n+1}}$

then $\left\{x_{n}\right\}$ converges strongly to $Q x$, where $Q$ is the sunny nonexpansive retraction of $E$ onto $F(T)=\bigcap_{i=1}^{\infty} F\left(T_{n}\right)$.

In 2005, Aoyama et al. [3] proved a weak convergence theorem for finding a solution of problem (1.3) as follows.

Theorem 1.6 Let E be a uniformly convex and 2-uniformly smooth Banach space and let $C$ be a nonempty closed convex subset of $E$. Let $Q_{C}$ be a sunny nonexpansive retraction from $E$ onto $C$, let $\alpha>0$ and let $A$ be an $\alpha$-inverse strongly accretive operator of $C$ into $E$ with $S(C, A) \neq \emptyset$. Suppose that $x_{1}=x \in C$ and $\left\{x_{n}\right\}$ is given by

$$
x_{n+1}=\alpha_{n} x_{n}+\left(1-\alpha_{n}\right) Q_{C}\left(x_{n}-\lambda_{n} A x_{n}\right)
$$

for every $n=1,2, \ldots$, where $\left\{\lambda_{n}\right\}$ is a sequence of positive real numbers and $\left\{\alpha_{n}\right\}$ is a sequence in $[0,1]$. If $\left\{\lambda_{n}\right\}$ and $\left\{\alpha_{n}\right\}$ are chosen so that $\lambda_{n} \in\left[a, \frac{\alpha}{K^{2}}\right]$ for some $a>0$ and $\alpha_{n} \in[b, c]$ for some $b, c$ with $0<b<c<1$, then $\left\{x_{n}\right\}$ converges weakly to some element $z$ of $S(C, A)$, where $K$ is the 2-uniformly smoothness constant of $E$.

In 2009, Kangtunykarn and Suantai [12] introduced the $S$-mapping generated by a finite family of mappings and real numbers as follows.

Definition 1.4 Let $C$ be a nonempty convex subset of a real Banach space. Let $\left\{T_{i}\right\}_{i=1}^{N}$ be a finite family of mappings of $C$ into itself. For each $j=1,2, \ldots, N$, let $\alpha_{j}=\left(\alpha_{1}^{j}, \alpha_{2}^{j}, \alpha_{3}^{j}\right) \in$ $I \times I \times I$, where $I \in[0,1]$ and $\alpha_{1}^{j}+\alpha_{2}^{j}+\alpha_{3}^{j}=1$. Define the mapping $S: C \rightarrow C$ as follows:

$$
\begin{aligned}
& U_{0}=I, \\
& U_{1}=\alpha_{1}^{1} T_{1} U_{0}+\alpha_{2}^{1} U_{0}+\alpha_{3}^{1} I, \\
& U_{2}=\alpha_{1}^{2} T_{2} U_{1}+\alpha_{2}^{2} U_{1}+\alpha_{3}^{2} I, \\
& U_{3}=\alpha_{1}^{3} T_{3} U_{2}+\alpha_{2}^{3} U_{2}+\alpha_{3}^{3} I, \\
& \vdots \\
& U_{N-1}=\alpha_{1}^{N-1} T_{N-1} U_{N-2}+\alpha_{2}^{N-1} U_{N-2}+\alpha_{3}^{N-1} I, \\
& S=U_{N}=\alpha_{1}^{N} T_{N} U_{N-1}+\alpha_{2}^{N} U_{N-1}+\alpha_{3}^{N} I .
\end{aligned}
$$

This mapping is called the $S$-mapping generated by $T_{1}, T_{2}, \ldots, T_{N}$ and $\alpha_{1}, \alpha_{2}, \ldots, \alpha_{N}$.

For every $i=1,2, \ldots, N$, put $\alpha_{3}^{j}=0$ in (1.7), then the $S$-mapping generated by $T_{1}, T_{2}$, $\ldots, T_{N}$ and $\alpha_{1}, \alpha_{2}, \ldots, \alpha_{N}$ reduces to the $K$-mapping generated by $T_{1}, T_{2}, \ldots, T_{N}$ and $\alpha_{1}^{1}, \alpha_{1}^{2}, \ldots, \alpha_{1}^{N}$, which is defined by Kangtunyakarn and Suantai [13]. 
Recently, Kangtunyakarn [14] introduced an iterative scheme by the modification of Mann's iteration process for finding a common element of the set of solutions of a finite family of variational inequality problems and the set of fixed points of an $\eta$-strictly pseudocontractive mapping and a nonexpansive mapping as follows.

Theorem 1.7 Let $C$ be a nonempty closed convex subset of a uniformly convex and 2-uniformly smooth Banach space $E$. Let $Q_{C}$ be the sunny nonexpansive retraction from $E$ onto $C$. For every $i=1,2, \ldots, N$, let $A_{i}: C \rightarrow E$ be an $\alpha_{i}$-inverse strongly accretive mapping. Define a mapping $G_{i}: C \rightarrow C$ by $Q_{C}\left(I-\lambda_{i} A_{i}\right) x=G_{i} x$ for all $x \in C$ and $i=1,2, \ldots, N$, where $\lambda_{i} \in\left(0, \frac{\alpha_{i}}{K^{2}}\right), K$ is the 2-uniformly smooth constant of $E$. Let $B: C \rightarrow C$ be the $K$-mapping generated by $G_{1}, G_{2}, \ldots, G_{N}$ and $\rho_{1}, \rho_{2}, \ldots, \rho_{N}$, where $\rho_{i} \in(0,1), \forall i=1,2, \ldots, N-1$ and $\rho_{N} \in(0,1]$. Let $T: C \rightarrow C$ be a nonexpansive mapping and $S: C \rightarrow C$ be an $\eta$-strictly pseudo-contractive mapping with $\mathcal{F}=F(S) \cap F(T) \cap \bigcap_{i=1}^{N} S\left(C, A_{i}\right) \neq \emptyset$. Define a mapping $B_{A}: C \rightarrow C$ by $T((1-\alpha) I+\alpha S) x=B_{A} x, \forall x \in C$ and $\alpha \in\left(0, \frac{\eta}{K^{2}}\right)$. Let $\left\{x_{n}\right\}$ be a sequence generated by $x_{1} \in C$ and

$$
x_{n+1}=\alpha_{n} f\left(x_{n}\right)+\beta_{n} x_{n}+\gamma_{n} B x_{n}+\delta_{n} B_{A} x_{n}, \quad \forall n \geq 1,
$$

where $f: C \rightarrow C$ is a contractive mapping and $\left\{\alpha_{n}\right\},\left\{\beta_{n}\right\},\left\{\gamma_{n}\right\},\left\{\delta_{n}\right\} \subseteq[0,1], \alpha_{n}+\beta_{n}+\gamma_{n}+$ $\delta_{n}=1$ and satisfy the following conditions:

(i) $\lim _{n \rightarrow \infty} \alpha_{n}=0$ and $\sum_{n=1}^{\infty} \alpha_{n}=\infty$,

(ii) $\left\{\gamma_{n}\right\},\left\{\delta_{n}\right\} \subseteq[c, d] \subset(0,1)$ for some $c, d>0$ and $\forall n \geq 1$,

(iii) $\quad \sum_{n=1}^{\infty}\left|\beta_{n+1}-\beta_{n}\right|, \quad \sum_{n=1}^{\infty}\left|\gamma_{n+1}-\gamma_{n}\right|, \quad \sum_{n=1}^{\infty}\left|\delta_{n+1}-\delta_{n}\right|<\infty$,

(iv) $0<\liminf _{n \rightarrow \infty} \beta_{n} \leq \limsup _{n \rightarrow \infty} \beta_{n}<1$.

Then the sequence $\left\{x_{n}\right\}$ converses strongly to $q \in \mathcal{F}$, which solves the following variational inequality:

$$
\langle q-f(q), j(q-p)\rangle \leq 0, \quad \forall p \in \mathcal{F} .
$$

Question How can we prove a strong convergence theorem for the set of fixed points of a finite family of nonexpansive mappings and the set of fixed points of a finite family of strictly pseudo-contractive mappings and the set of solutions of variational inequality problems in a uniformly convex and 2-uniformly smooth Banach space?

Motivated by the $S$-mapping, we define a new mapping in the next section to answer the above question, and from Theorems 1.4, 1.5, 1.6 and 1.7 we modify the Halpern iteration for finding a common element of two sets of solutions of (1.3) and the set of fixed points of a finite family of nonexpansive mappings and the set of fixed points of a finite family of strictly pseudo-contractive mappings in a uniformly convex and 2-uniformly smooth Banach space. Moreover, by using our main result, we also obtain a strong convergence theorem for a finite family of the set of solutions of (1.3) and the set of fixed points of a finite family of strictly pseudo-contractive mappings. 


\section{Preliminaries}

In this section we collect and prove the following lemmas to use in our main result.

Lemma 2.1 (See [15]) Let E be a real 2-uniformly smooth Banach space with the best smooth constant $K$. Then the following inequality holds:

$$
\|x+y\|^{2} \leq\|x\|^{2}+2\langle y, J(x)\rangle+2\|K y\|^{2}
$$

for any $x, y \in E$.

Lemma 2.2 (See [16]) Let $X$ be a uniformly convex Banach space and $B_{r}=\{x \in X:\|x\| \leq$ $r\}, r>0$. Then there exists a continuous, strictly increasing and convex function $g:[0, \infty] \rightarrow$ $[0, \infty], g(0)=0$ such that

$$
\|\alpha x+\beta y+\gamma z\|^{2} \leq \alpha\|x\|^{2}+\beta\|y\|^{2}+\gamma\|z\|^{2}-\alpha \beta g(\|x-y\|)
$$

for all $x, y, z \in B_{r}$ and all $\alpha, \beta, \gamma \in[0,1]$ with $\alpha+\beta+\gamma=1$.

Lemma 2.3 (See [3]) Let $C$ be a nonempty closed convex subset of a smooth Banach space $E$. Let $Q_{C}$ be a sunny nonexpansive retraction from $E$ onto $C$ and let $A$ be an accretive operator of $C$ into $E$. Then, for all $\lambda>0$,

$$
S(C, A)=F\left(Q_{C}(I-\lambda A)\right) .
$$

Lemma 2.4 (See [15]) Let $r>0$. If $E$ is uniformly convex, then there exists a continuous, strictly increasing and convex function $g:[0, \infty) \rightarrow[0, \infty), g(0)=0$ such that for all $x, y \in$ $B_{r}(0)=\{x \in E:\|x\| \leq r\}$ and for any $\alpha \in[0,1]$, we have $\|\alpha x+(1-\alpha) y\|^{2} \leq \alpha\|x\|^{2}+(1-$ $\alpha)\|y\|^{2}-\alpha(1-\alpha) g(\|x-y\|)$.

Lemma 2.5 (See [17]) Let $C$ be a closed and convex subset of a real uniformly smooth Banach space $E$ and let $T: C \rightarrow C$ be a nonexpansive mapping with a nonempty fixed point $F(T)$. If $\left\{x_{n}\right\} \subset C$ is a bounded sequence such that $\lim _{n \rightarrow \infty}\left\|x_{n}-T x_{n}\right\|=0$. Then there exists a unique sunny nonexpansive retraction $Q_{F(T)}: C \rightarrow F(T)$ such that

$$
\limsup _{n \rightarrow \infty}\left\langle u-Q_{F(T)} u, J\left(x_{n}-Q_{F(T)} u\right)\right\rangle \leq 0
$$

for any given $u \in C$.

Lemma 2.6 (See [18]) Let $\left\{s_{n}\right\}$ be a sequence of nonnegative real numbers satisfying

$$
s_{n+1}=\left(1-\alpha_{n}\right) s_{n}+\delta_{n}, \quad \forall n \geq 0,
$$

where $\left\{\alpha_{n}\right\}$ is a sequence in $(0,1)$ and $\left\{\delta_{n}\right\}$ is a sequence such that

$$
\text { (1) } \sum_{n=1}^{\infty} \alpha_{n}=\infty \text {, }
$$


(2) $\quad \limsup _{n \rightarrow \infty} \frac{\delta_{n}}{\alpha_{n}} \leq 0 \quad$ or $\quad \sum_{n=1}^{\infty}\left|\delta_{n}\right|<\infty$.

Then $\lim _{n \rightarrow \infty} s_{n}=0$.

From the $S$-mapping, we define the mapping generated by two sets of finite families of the mappings and real numbers as follows.

Definition 2.1 Let $C$ be a nonempty convex subset of a Banach space. Let $\left\{S_{i}\right\}_{i=1}^{N}$ and $\left\{T_{i}\right\}_{i=1}^{N}$ be two finite families of mappings of $C$ into itself. For each $j=1,2, \ldots, N$, let $\alpha_{j}=$ $\left(\alpha_{1}^{j}, \alpha_{2}^{j}, \alpha_{3}^{j}\right) \in I \times I \times I$, where $I \in[0,1]$ and $\alpha_{1}^{j}+\alpha_{2}^{j}+\alpha_{3}^{j}=1$. We define the mapping $S^{A}$ : $C \rightarrow C$ as follows:

$$
\begin{aligned}
& U_{0}=T_{1}=I \\
& U_{1}=T_{1}\left(\alpha_{1}^{1} S_{1} U_{0}+\alpha_{2}^{1} U_{0}+\alpha_{3}^{1} I\right), \\
& U_{2}=T_{2}\left(\alpha_{1}^{2} S_{2} U_{1}+\alpha_{2}^{2} U_{1}+\alpha_{3}^{2} I\right), \\
& U_{3}=T_{3}\left(\alpha_{1}^{3} S_{3} U_{2}+\alpha_{2}^{3} U_{2}+\alpha_{3}^{3} I\right), \\
& \vdots \\
& U_{N-1}=T_{N-1}\left(\alpha_{1}^{N-1} S_{N-1} U_{N-2}+\alpha_{2}^{N-1} U_{N-2}+\alpha_{3}^{N-1} I\right), \\
& S^{A}=U_{N}=T_{N}\left(\alpha_{1}^{N} S_{N} U_{N-1}+\alpha_{2}^{N} U_{N-1}+\alpha_{3}^{N} I\right) .
\end{aligned}
$$

This mapping is called the $S^{A}$-mapping generated by $S_{1}, S_{2}, \ldots, S_{N}, T_{1}, T_{2}, \ldots, T_{N}$ and $\alpha_{1}, \alpha_{2}, \ldots, \alpha_{N}$.

Lemma 2.7 Let $C$ be a nonempty closed convex subset of a 2-uniformly smooth and uniformly convex Banach space. Let $\left\{S_{i}\right\}_{i=1}^{N}$ be a finite family of $\kappa_{i}$-strict pseudo-contractions of $C$ into itself and let $\left\{T_{i}\right\}_{i=1}^{N}$ be a finite family of nonexpansive mappings of $C$ into itself with $\bigcap_{i=1}^{N} F\left(S_{i}\right) \cap \bigcap_{i=1}^{N} F\left(T_{i}\right) \neq \emptyset$ and $\kappa=\min \left\{\kappa_{i}: i=1,2, \ldots, N\right\}$ with $K^{2} \leq \kappa$, where $K$ is the 2-uniformly smooth constant of E. Let $\alpha_{j}=\left(\alpha_{1}^{j}, \alpha_{2}^{j}, \alpha_{3}^{j}\right) \in I \times I \times I$, where $I=[0,1]$, $\alpha_{1}^{j}+\alpha_{2}^{j}+\alpha_{3}^{j}=1, \alpha_{1}^{j} \in(0,1], \alpha_{2}^{j} \in[0,1]$ and $\alpha_{3}^{j} \in(0,1)$ for all $j=1,2, \ldots, N$. Let $S^{A}$ be the $S^{A}$-mapping generated by $S_{1}, S_{2}, \ldots, S_{N}, T_{1}, T_{2}, \ldots, T_{N}$ and $\alpha_{1}, \alpha_{2}, \ldots, \alpha_{N}$. Then $F\left(S^{A}\right)=$ $\bigcap_{i=1}^{N} F\left(S_{i}\right) \cap \bigcap_{i=1}^{N} F\left(T_{i}\right)$ and $S^{A}$ is a nonexpansive mapping.

Proof Let $x_{0} \in F\left(S^{A}\right)$ and $x^{*} \in \bigcap_{i=1}^{N} F\left(S_{i}\right) \cap \bigcap_{i=1}^{N} F\left(T_{i}\right)$, we have

$$
\begin{aligned}
\left\|x_{0}-x^{*}\right\|^{2}= & \left\|T_{N}\left(\alpha_{1}^{N} S_{N} U_{N-1}+\alpha_{2}^{N} U_{N-1}+\alpha_{3}^{N} I\right) x_{0}-x^{*}\right\|^{2} \\
\leq & \left\|\alpha_{1}^{N}\left(S_{N} U_{N-1} x_{0}-x^{*}\right)+\alpha_{2}^{N}\left(U_{N-1} x_{0}-x^{*}\right)+\alpha_{3}^{N}\left(x_{0}-x^{*}\right)\right\|^{2} \\
= & \|\left(1-\alpha_{3}^{N}\right)\left(\frac{\alpha_{1}^{N}}{1-\alpha_{3}^{N}}\left(S_{N} U_{N-1} x_{0}-x^{*}\right)+\frac{\alpha_{2}^{N}}{1-\alpha_{3}^{N}}\left(U_{N-1} x_{0}-x^{*}\right)\right) \\
& +\alpha_{3}^{N}\left(x_{0}-x^{*}\right) \|^{2} \\
& \left(1-\alpha_{3}^{N}\right)\left\|\frac{\alpha_{1}^{N}}{1-\alpha_{3}^{N}}\left(S_{N} U_{N-1} x_{0}-x^{*}\right)+\frac{\alpha_{2}^{N}}{1-\alpha_{3}^{N}}\left(U_{N-1} x_{0}-x^{*}\right)\right\|^{2}
\end{aligned}
$$




$$
\begin{aligned}
& +\alpha_{3}^{N}\left\|x_{0}-x^{*}\right\|^{2} \\
& =\left(1-\alpha_{3}^{N}\right)\left\|\frac{\alpha_{1}^{N}}{1-\alpha_{3}^{N}}\left(S_{N} U_{N-1} x_{0}-x^{*}\right)+\left(1-\frac{\alpha_{1}^{N}}{1-\alpha_{3}^{N}}\right)\left(U_{N-1} x_{0}-x^{*}\right)\right\|^{2} \\
& +\alpha_{3}^{N}\left\|x_{0}-x^{*}\right\|^{2} \\
& =\left(1-\alpha_{3}^{N}\right)\left\|\frac{\alpha_{1}^{N}}{1-\alpha_{3}^{N}}\left(S_{N} U_{N-1} x_{0}-U_{N-1} x_{0}\right)+U_{N-1} x_{0}-x^{*}\right\|^{2}+\alpha_{3}^{N}\left\|x_{0}-x^{*}\right\|^{2} \\
& \leq\left(1-\alpha_{3}^{N}\right)\left(\left\|U_{N-1} x_{0}-x^{*}\right\|^{2}\right. \\
& +2 \frac{\alpha_{1}^{N}}{1-\alpha_{3}^{N}}\left\langle S_{N} U_{N-1} x_{0}-U_{N-1} x_{0}, j\left(U_{N-1} x_{0}-x^{*}\right)\right\rangle \\
& \left.+2 K^{2}\left(\frac{\alpha_{1}^{N}}{1-\alpha_{3}^{N}}\right)^{2}\left\|S_{N} U_{N-1} x_{0}-U_{N-1} x_{0}\right\|^{2}\right)+\alpha_{3}^{N}\left\|x_{0}-x^{*}\right\|^{2} \\
& =\left(1-\alpha_{3}^{N}\right)\left(\left\|U_{N-1} x_{0}-x^{*}\right\|^{2}+2 \frac{\alpha_{1}^{N}}{1-\alpha_{3}^{N}}\left\langle S_{N} U_{N-1} x_{0}-x^{*}, j\left(U_{N-1} x_{0}-x^{*}\right)\right\rangle\right. \\
& +2 \frac{\alpha_{1}^{N}}{1-\alpha_{3}^{N}}\left\langle x^{*}-U_{N-1} x_{0}, j\left(U_{N-1} x_{0}-x^{*}\right)\right\rangle \\
& \left.+2 K^{2}\left(\frac{\alpha_{1}^{N}}{1-\alpha_{3}^{N}}\right)^{2}\left\|S_{N} U_{N-1} x_{0}-U_{N-1} x_{0}\right\|^{2}\right)+\alpha_{3}^{N}\left\|x_{0}-x^{*}\right\|^{2} \\
& \leq\left(1-\alpha_{3}^{N}\right)\left(\left\|U_{N-1} x_{0}-x^{*}\right\|^{2}\right. \\
& +2 \frac{\alpha_{1}^{N}}{1-\alpha_{3}^{N}}\left(\left\|U_{N-1} x_{0}-x^{*}\right\|^{2}-\kappa\left\|\left(I-S_{N}\right) U_{N-1} x_{0}\right\|^{2}\right) \\
& \left.-2 \frac{\alpha_{1}^{N}}{1-\alpha_{3}^{N}}\left\|x^{*}-U_{N-1} x_{0}\right\|^{2}+2 K^{2}\left(\frac{\alpha_{1}^{N}}{1-\alpha_{3}^{N}}\right)^{2}\left\|S_{N} U_{N-1} x_{0}-U_{N-1} x_{0}\right\|^{2}\right) \\
& +\alpha_{3}^{N}\left\|x_{0}-x^{*}\right\|^{2} \\
& =\left(1-\alpha_{3}^{N}\right)\left(\left\|U_{N-1} x_{0}-x^{*}\right\|^{2}-2 \frac{\alpha_{1}^{N}}{1-\alpha_{3}^{N}} \kappa\left\|\left(I-S_{N}\right) U_{N-1} x_{0}\right\|^{2}\right. \\
& \left.+2 K^{2}\left(\frac{\alpha_{1}^{N}}{1-\alpha_{3}^{N}}\right)^{2}\left\|S_{N} U_{N-1} x_{0}-U_{N-1} x_{0}\right\|^{2}\right)+\alpha_{3}^{N}\left\|x_{0}-x^{*}\right\|^{2} \\
& =\left(1-\alpha_{3}^{N}\right)\left(\left\|U_{N-1} x_{0}-x^{*}\right\|^{2}\right. \\
& \left.-2 \frac{\alpha_{1}^{N}}{1-\alpha_{3}^{N}}\left(\kappa-K^{2}\left(\frac{\alpha_{1}^{N}}{1-\alpha_{3}^{N}}\right)\right)\left\|\left(I-S_{N}\right) U_{N-1} x_{0}\right\|^{2}\right)+\alpha_{3}^{N}\left\|x_{0}-x^{*}\right\|^{2} \\
& \leq\left(1-\alpha_{3}^{N}\right)\left\|U_{N-1} x_{0}-x^{*}\right\|^{2}+\alpha_{3}^{N}\left\|x_{0}-x^{*}\right\|^{2} \\
& \leq\left(1-\alpha_{3}^{N}\right)\left(\left(1-\alpha_{3}^{N-1}\right)\left\|U_{N-2} x_{0}-x^{*}\right\|^{2}+\alpha_{3}^{N-1}\left\|x_{0}-x^{*}\right\|^{2}\right)+\alpha_{3}^{N}\left\|x_{0}-x^{*}\right\|^{2} \\
& =\prod_{j=N-1}^{N}\left(1-\alpha_{3}^{j}\right)\left\|U_{N-2} x_{0}-x^{*}\right\|^{2}+\left(1-\prod_{j=N-1}^{N}\left(1-\alpha_{3}^{j}\right)\right)\left\|x_{0}-x^{*}\right\|^{2}
\end{aligned}
$$


Kangtunyakarn Fixed Point Theory and Applications 2013, 2013:157

Page 10 of 24

http://www.fixedpointtheoryandapplications.com/content/2013/1/157

$$
\begin{aligned}
& \leq \prod_{j=3}^{N}\left(1-\alpha_{3}^{j}\right)\left\|U_{2} x_{0}-x^{*}\right\|^{2}+\left(1-\prod_{j=3}^{N}\left(1-\alpha_{3}^{j}\right)\right)\left\|x_{0}-x^{*}\right\|^{2} \\
& =\prod_{j=3}^{N}\left(1-\alpha_{3}^{j}\right)\left\|T_{2}\left(\alpha_{1}^{2} S_{2} U_{1}+\alpha_{2}^{2} U_{1}+\alpha_{3}^{2} I\right) x_{0}-x^{*}\right\|^{2} \\
& +\left(1-\prod_{j=3}^{N}\left(1-\alpha_{3}^{j}\right)\right)\left\|x_{0}-x^{*}\right\|^{2} \\
& \leq \prod_{j=3}^{N}\left(1-\alpha_{3}^{j}\right)\left\|\alpha_{1}^{2}\left(S_{2} U_{1} x_{0}-x^{*}\right)+\alpha_{2}^{2}\left(U_{1} x_{0}-x^{*}\right)+\alpha_{3}^{2}\left(x_{0}-x^{*}\right)\right\|^{2} \\
& +\left(1-\prod_{j=3}^{N}\left(1-\alpha_{3}^{j}\right)\right)\left\|x_{0}-x^{*}\right\|^{2} \\
& =\prod_{j=3}^{N}\left(1-\alpha_{3}^{j}\right) \|\left(1-\alpha_{3}^{2}\right)\left(\frac{\alpha_{1}^{2}}{1-\alpha_{3}^{2}}\left(S_{2} U_{1} x_{0}-x^{*}\right)+\frac{\alpha_{2}^{2}}{1-\alpha_{3}^{2}}\left(U_{1} x_{0}-x^{*}\right)\right) \\
& +\alpha_{3}^{2}\left(x_{0}-x^{*}\right)\left\|^{2}+\left(1-\prod_{j=3}^{N}\left(1-\alpha_{3}^{j}\right)\right)\right\| x_{0}-x^{*} \|^{2} \\
& \leq \prod_{j=3}^{N}\left(1-\alpha_{3}^{j}\right)\left(\left(1-\alpha_{3}^{2}\right) \| \frac{\alpha_{1}^{2}}{1-\alpha_{3}^{2}}\left(S_{2} U_{1} x_{0}-x^{*}\right)\right. \\
& \left.+\frac{\alpha_{2}^{2}}{1-\alpha_{3}^{2}}\left(U_{1} x_{0}-x^{*}\right)\left\|^{2}+\alpha_{3}^{2}\right\| x_{0}-x^{*} \|^{2}\right)+\left(1-\prod_{j=3}^{N}\left(1-\alpha_{3}^{j}\right)\right)\left\|x_{0}-x^{*}\right\|^{2} \\
& =\prod_{j=3}^{N}\left(1-\alpha_{3}^{j}\right)\left(\left(1-\alpha_{3}^{2}\right)\left\|\frac{\alpha_{1}^{2}}{1-\alpha_{3}^{2}}\left(S_{2} U_{1} x_{0}-x^{*}\right)+\left(1-\frac{\alpha_{1}^{2}}{1-\alpha_{3}^{2}}\right)\left(U_{1} x_{0}-x^{*}\right)\right\|^{2}\right. \\
& \left.+\alpha_{3}^{2}\left\|x_{0}-x^{*}\right\|^{2}\right)+\left(1-\prod_{j=3}^{N}\left(1-\alpha_{3}^{j}\right)\right)\left\|x_{0}-x^{*}\right\|^{2} \\
& =\prod_{j=3}^{N}\left(1-\alpha_{3}^{j}\right)\left(\left(1-\alpha_{3}^{2}\right)\left\|\frac{\alpha_{1}^{2}}{1-\alpha_{3}^{2}}\left(S_{2} U_{1} x_{0}-U_{1} x_{0}\right)+U_{1} x_{0}-x^{*}\right\|^{2}\right. \\
& \left.+\alpha_{3}^{2}\left\|x_{0}-x^{*}\right\|^{2}\right)+\left(1-\prod_{j=3}^{N}\left(1-\alpha_{3}^{j}\right)\right)\left\|x_{0}-x^{*}\right\|^{2} \\
& \leq \prod_{j=3}^{N}\left(1-\alpha_{3}^{j}\right)\left(( 1 - \alpha _ { 3 } ^ { 2 } ) \left(\left\|U_{1} x_{0}-x^{*}\right\|^{2}\right.\right. \\
& +2 \frac{\alpha_{1}^{2}}{1-\alpha_{3}^{2}}\left\langle S_{2} U_{1} x_{0}-U_{1} x_{0}, j\left(U_{1} x_{0}-x^{*}\right)\right\rangle \\
& \left.\left.+2 K^{2}\left(\frac{\alpha_{1}^{2}}{1-\alpha_{3}^{2}}\right)\left\|S_{2} U_{1} x_{0}-U_{1} x_{0}\right\|^{2}\right)+\alpha_{3}^{2}\left\|x_{0}-x^{*}\right\|^{2}\right) \\
& +\left(1-\prod_{j=3}^{N}\left(1-\alpha_{3}^{j}\right)\right)\left\|x_{0}-x^{*}\right\|^{2}
\end{aligned}
$$


Kangtunyakarn Fixed Point Theory and Applications 2013, 2013:157

Page 11 of 24

http://www.fixedpointtheoryandapplications.com/content/2013/1/157

$$
\begin{aligned}
& \leq \prod_{j=3}^{N}\left(1-\alpha_{3}^{j}\right)\left(( 1 - \alpha _ { 3 } ^ { 2 } ) \left(\left\|U_{1} x_{0}-x^{*}\right\|^{2}\right.\right. \\
& \left.-2 \frac{\alpha_{1}^{2}}{1-\alpha_{3}^{2}}\left(\kappa-K^{2}\left(\frac{\alpha_{1}^{2}}{1-\alpha_{3}^{2}}\right)\right)\left\|\left(I-S_{2}\right) U_{1} x_{0}\right\|^{2}\right) \\
& \left.+\alpha_{3}^{2}\left\|x_{0}-x^{*}\right\|^{2}\right)+\left(1-\prod_{j=3}^{N}\left(1-\alpha_{3}^{j}\right)\right)\left\|x_{0}-x^{*}\right\|^{2} \\
& \leq \prod_{j=3}^{N}\left(1-\alpha_{3}^{j}\right)\left(1-\alpha_{3}^{2}\right)\left(\left\|U_{1} x_{0}-x^{*}\right\|^{2}+\alpha_{3}^{2}\left\|x_{0}-x^{*}\right\|^{2}\right) \\
& +\left(1-\prod_{j=3}^{N}\left(1-\alpha_{3}^{j}\right)\right)\left\|x_{0}-x^{*}\right\|^{2} \\
& =\prod_{j=2}^{N}\left(1-\alpha_{3}^{j}\right)\left\|U_{1} x_{0}-x^{*}\right\|^{2}+\left(1-\prod_{j=2}^{N}\left(1-\alpha_{3}^{j}\right)\right)\left\|x_{0}-x^{*}\right\|^{2} \\
& =\prod_{j=2}^{N}\left(1-\alpha_{3}^{j}\right)\left\|\alpha_{1}^{1}\left(S_{1} U_{0} x_{0}-x^{*}\right)+\alpha_{2}^{1}\left(U_{0} x_{0}-x^{*}\right)+\alpha_{3}^{1}\left(x_{0}-x^{*}\right)\right\|^{2} \\
& +\left(1-\prod_{j=2}^{N}\left(1-\alpha_{3}^{j}\right)\right)\left\|x_{0}-x^{*}\right\|^{2} \\
& =\prod_{j=2}^{N}\left(1-\alpha_{3}^{j}\right)\left\|\alpha_{1}^{1}\left(S_{1} x_{0}-x^{*}\right)+\left(1-\alpha_{1}^{1}\right)\left(x_{0}-x^{*}\right)\right\|^{2} \\
& +\left(1-\prod_{j=2}^{N}\left(1-\alpha_{3}^{j}\right)\right)\left\|x_{0}-x^{*}\right\|^{2} \\
& =\prod_{j=2}^{N}\left(1-\alpha_{3}^{j}\right)\left\|\alpha_{1}^{1}\left(S_{1} x_{0}-x_{0}\right)+x_{0}-x^{*}\right\|^{2}+\left(1-\prod_{j=2}^{N}\left(1-\alpha_{3}^{j}\right)\right)\left\|x_{0}-x^{*}\right\|^{2} \\
& \leq \prod_{j=2}^{N}\left(1-\alpha_{3}^{j}\right)\left(\left\|x_{0}-x^{*}\right\|^{2}+2 \alpha_{1}^{1}\left\langle S_{1} x_{0}-x_{0}, j\left(x_{0}-x^{*}\right)\right\rangle\right. \\
& \left.+2 K^{2}\left(\alpha_{1}^{1}\right)^{2}\left\|S_{1} x_{0}-x_{0}\right\|^{2}\right)+\left(1-\prod_{j=2}^{N}\left(1-\alpha_{3}^{j}\right)\right)\left\|x_{0}-x^{*}\right\|^{2} \\
& =\prod_{j=2}^{N}\left(1-\alpha_{3}^{j}\right)\left(\left\|x_{0}-x^{*}\right\|^{2}+2 \alpha_{1}^{1}\left\langle S_{1} x_{0}-x^{*}, j\left(x_{0}-x^{*}\right)\right\rangle\right. \\
& +2 \alpha_{1}^{1}\left\langle x^{*}-x_{0}, j\left(x_{0}-x^{*}\right)\right\rangle \\
& \left.+2 K^{2}\left(\alpha_{1}^{1}\right)^{2}\left\|S_{1} x_{0}-x_{0}\right\|^{2}\right)+\left(1-\prod_{j=2}^{N}\left(1-\alpha_{3}^{j}\right)\right)\left\|x_{0}-x^{*}\right\|^{2} \\
& \leq \prod_{j=2}^{N}\left(1-\alpha_{3}^{j}\right)\left(\left\|x_{0}-x^{*}\right\|^{2}+2 \alpha_{1}^{1}\left(\left\|x_{0}-x^{*}\right\|-\kappa\left\|S_{1} x_{0}-x_{0}\right\|^{2}\right)\right. \\
& -2 \alpha_{1}^{1}\left\|x^{*}-x_{0}\right\|^{2}
\end{aligned}
$$


Kangtunyakarn Fixed Point Theory and Applications 2013, 2013:157

Page 12 of 24

$$
\begin{aligned}
& \left.+2 K^{2}\left(\alpha_{1}^{1}\right)^{2}\left\|S_{1} x_{0}-x_{0}\right\|^{2}\right)+\left(1-\prod_{j=2}^{N}\left(1-\alpha_{3}^{j}\right)\right)\left\|x_{0}-x^{*}\right\|^{2} \\
= & \prod_{j=2}^{N}\left(1-\alpha_{3}^{j}\right)\left(\left\|x_{0}-x^{*}\right\|^{2}-2 \alpha_{1}^{1}\left(\kappa-K^{2} \alpha_{1}^{1}\right)\left\|S_{1} x_{0}-x_{0}\right\|^{2}\right) \\
& +\left(1-\prod_{j=2}^{N}\left(1-\alpha_{3}^{j}\right)\right)\left\|x_{0}-x^{*}\right\|^{2} \\
= & \left\|x_{0}-x^{*}\right\|^{2}-\prod_{j=2}^{N}\left(1-\alpha_{3}^{j}\right) 2 \alpha_{1}^{1}\left(\kappa-K^{2} \alpha_{1}^{1}\right)\left\|S_{1} x_{0}-x_{0}\right\|^{2} \\
\leq & \left\|x_{0}-x^{*}\right\|^{2} .
\end{aligned}
$$

For every $j=1,2, \ldots, N$ and (2.2), we have

$$
\left\|U_{j} x_{0}-x^{*}\right\|^{2} \leq\left\|x_{0}-x^{*}\right\|^{2} .
$$

For every $k=1,2, \ldots, N-1$ and (2.2) we have

$$
\begin{aligned}
\left\|x_{0}-x^{*}\right\|^{2} \leq & \prod_{j=k+1}^{N}\left(1-\alpha_{3}^{j}\right)\left\|U_{k} x_{0}-x^{*}\right\|^{2}+\left(1-\prod_{j=k+1}^{N}\left(1-\alpha_{3}^{j}\right)\right)\left\|x_{0}-x^{*}\right\|^{2} \\
= & \prod_{j=k+1}^{N}\left(1-\alpha_{3}^{j}\right)\left\|T_{k}\left(\alpha_{1}^{k} S_{k} U_{k-1}+\alpha_{2}^{k} U_{k-1}+\alpha_{3}^{k} I\right) x_{0}-x^{*}\right\|^{2} \\
& +\left(1-\prod_{j=k+1}^{N}\left(1-\alpha_{3}^{j}\right)\right)\left\|x_{0}-x^{*}\right\|^{2} \\
\leq & \prod_{j=k+1}^{N}\left(1-\alpha_{3}^{j}\right)\left\|\alpha_{1}^{k}\left(S_{k} U_{k-1} x_{0}-x^{*}\right)+\alpha_{2}^{k}\left(U_{k-1} x_{0}-x^{*}\right)+\alpha_{3}^{k}\left(x_{0}-x^{*}\right)\right\|^{2} \\
& +\left(1-\prod_{j=k+1}^{N}\left(1-\alpha_{3}^{j}\right)\right)\left\|x_{0}-x^{*}\right\|^{2} \\
= & \prod_{j=k+1}^{N}\left(1-\alpha_{3}^{j}\right) \|\left(1-\alpha_{3}^{k}\right)\left(\frac{\alpha_{1}^{k}}{1-\alpha_{3}^{k}}\left(S_{k} U_{k-1} x_{0}-x^{*}\right)+\frac{\alpha_{2}^{k}}{1-\alpha_{3}^{k}}\left(U_{k-1} x_{0}-x^{*}\right)\right) \\
& +\alpha_{3}^{k}\left(x_{0}-x^{*}\right)\left\|^{2}+\left(1-\prod_{j=k+1}^{N}\left(1-\alpha_{3}^{j}\right)\right)\right\| x_{0}-x^{*} \|^{2} \\
\leq & \prod_{j=k+1}^{N}\left(1-\alpha_{3}^{j}\right)\left(\left(1-\alpha_{3}^{k}\right)\left\|\frac{\alpha_{1}^{k}}{1-\alpha_{3}^{k}}\left(S_{k} U_{k-1} x_{0}-x^{*}\right)+\frac{\alpha_{2}^{k}}{1-\alpha_{3}^{k}}\left(U_{k-1} x_{0}-x^{*}\right)\right\|^{2}\right. \\
& \left.+\alpha_{3}^{k}\left\|x_{0}-x^{*}\right\|^{2}\right)+\left(1-\prod_{j=k+1}^{N}\left(1-\alpha_{3}^{j}\right)\right)\left\|x_{0}-x^{*}\right\|^{2} \\
= & \prod_{j=k+1}^{N}\left(1-\alpha_{3}^{j}\right)\left(\left(1-\alpha_{3}^{k}\right) \| \frac{\alpha_{1}^{k}}{1-\alpha_{3}^{k}}\left(S_{k} U_{k-1} x_{0}-x^{*}\right)\right. \\
&
\end{aligned}
$$


Kangtunyakarn Fixed Point Theory and Applications 2013, 2013:157

Page 13 of 24

http://www.fixedpointtheoryandapplications.com/content/2013/1/157

$$
\begin{aligned}
& +\left(1-\frac{\alpha_{1}^{k}}{1-\alpha_{3}^{k}}\right)\left(U_{k-1} x_{0}-x^{*}\right) \|^{2} \\
& \left.+\alpha_{3}^{k}\left\|x_{0}-x^{*}\right\|^{2}\right)+\left(1-\prod_{j=k+1}^{N}\left(1-\alpha_{3}^{j}\right)\right)\left\|x_{0}-x^{*}\right\|^{2} \\
& =\prod_{j=k+1}^{N}\left(1-\alpha_{3}^{j}\right)\left(\left(1-\alpha_{3}^{k}\right)\left\|\frac{\alpha_{1}^{k}}{1-\alpha_{3}^{k}}\left(S_{k} U_{k-1} x_{0}-U_{k-1} x_{0}\right)+U_{k-1} x_{0}-x^{*}\right\|^{2}\right. \\
& \left.+\alpha_{3}^{k}\left\|x_{0}-x^{*}\right\|^{2}\right)+\left(1-\prod_{j=k+1}^{N}\left(1-\alpha_{3}^{j}\right)\right)\left\|x_{0}-x^{*}\right\|^{2} \\
& \leq \prod_{j=k+1}^{N}\left(1-\alpha_{3}^{j}\right)\left(( 1 - \alpha _ { 3 } ^ { k } ) \left(\left\|U_{k-1} x_{0}-x^{*}\right\|^{2}\right.\right. \\
& +2 \frac{\alpha_{1}^{k}}{1-\alpha_{3}^{k}}\left\langle S_{k} U_{k-1} x_{0}-U_{k-1} x_{0}, j\left(U_{k-1} x_{0}-x^{*}\right)\right\rangle \\
& \left.\left.+2 K^{2}\left(\frac{\alpha_{1}^{k}}{1-\alpha_{3}^{k}}\right)^{2}\left\|S_{k} U_{k-1} x_{0}-U_{k-1} x_{0}\right\|^{2}\right)+\alpha_{3}^{k}\left\|x_{0}-x^{*}\right\|^{2}\right) \\
& +\left(1-\prod_{j=k+1}^{N}\left(1-\alpha_{3}^{j}\right)\right)\left\|x_{0}-x^{*}\right\|^{2} \\
& =\prod_{j=k+1}^{N}\left(1-\alpha_{3}^{j}\right)\left(( 1 - \alpha _ { 3 } ^ { k } ) \left(\left\|U_{k-1} x_{0}-x^{*}\right\|^{2}\right.\right. \\
& +2 \frac{\alpha_{1}^{k}}{1-\alpha_{3}^{k}}\left\langle S_{k} U_{k-1} x_{0}-x^{*}, j\left(U_{k-1} x_{0}-x^{*}\right)\right\rangle \\
& +2 \frac{\alpha_{1}^{k}}{1-\alpha_{3}^{k}}\left\langle x^{*}-U_{k-1} x_{0}, j\left(U_{k-1} x_{0}-x^{*}\right)\right\rangle \\
& \left.\left.+2 K^{2}\left(\frac{\alpha_{1}^{k}}{1-\alpha_{3}^{k}}\right)^{2}\left\|S_{k} U_{k-1} x_{0}-U_{k-1} x_{0}\right\|^{2}\right)+\alpha_{3}^{k}\left\|x_{0}-x^{*}\right\|^{2}\right) \\
& +\left(1-\prod_{j=k+1}^{N}\left(1-\alpha_{3}^{j}\right)\right)\left\|x_{0}-x^{*}\right\|^{2} \\
& \leq \prod_{j=k+1}^{N}\left(1-\alpha_{3}^{j}\right)\left(( 1 - \alpha _ { 3 } ^ { k } ) \left(\left\|U_{k-1} x_{0}-x^{*}\right\|^{2}\right.\right. \\
& +2 \frac{\alpha_{1}^{k}}{1-\alpha_{3}^{k}}\left(\left\|U_{k-1} x_{0}-x^{*}\right\|^{2}-\kappa\left\|\left(I-S_{k}\right) U_{k-1} x_{0}\right\|\right) \\
& -2 \frac{\alpha_{1}^{k}}{1-\alpha_{3}^{k}}\left\|x^{*}-U_{k-1} x_{0}\right\|^{2} \\
& \left.\left.+2 K^{2}\left(\frac{\alpha_{1}^{k}}{1-\alpha_{3}^{k}}\right)^{2}\left\|S_{k} U_{k-1} x_{0}-U_{k-1} x_{0}\right\|^{2}\right)+\alpha_{3}^{k}\left\|x_{0}-x^{*}\right\|^{2}\right) \\
& +\left(1-\prod_{j=k+1}^{N}\left(1-\alpha_{3}^{j}\right)\right)\left\|x_{0}-x^{*}\right\|^{2}
\end{aligned}
$$




$$
\begin{aligned}
= & \prod_{j=k+1}^{N}\left(1-\alpha_{3}^{j}\right)\left(( 1 - \alpha _ { 3 } ^ { k } ) \left(\left\|U_{k-1} x_{0}-x^{*}\right\|^{2}\right.\right. \\
& \left.\left.-2 \frac{\alpha_{1}^{k}}{1-\alpha_{3}^{k}}\left(\kappa-K^{2}\left(\frac{\alpha_{1}^{k}}{1-\alpha_{3}^{k}}\right)\right)\left\|\left(I-S_{k}\right) U_{k-1} x_{0}\right\|^{2}\right)+\alpha_{3}^{k}\left\|x_{0}-x^{*}\right\|^{2}\right) \\
& +\left(1-\prod_{j=k+1}^{N}\left(1-\alpha_{3}^{j}\right)\right)\left\|x_{0}-x^{*}\right\|^{2} \\
\leq & \prod_{j=k+1}^{N}\left(1-\alpha_{3}^{j}\right)\left(( 1 - \alpha _ { 3 } ^ { k } ) \left(\left\|x_{0}-x^{*}\right\|^{2}\right.\right. \\
& \left.\left.-2 \frac{\alpha_{1}^{k}}{1-\alpha_{3}^{k}}\left(\kappa-K^{2}\left(\frac{\alpha_{1}^{k}}{1-\alpha_{3}^{k}}\right)\right)\left\|\left(I-S_{k}\right) U_{k-1} x_{0}\right\|^{2}\right)+\alpha_{3}^{k}\left\|x_{0}-x^{*}\right\|^{2}\right) \\
& +\left(1-\prod_{j=k+1}^{N}\left(1-\alpha_{3}^{j}\right)\right)\left\|x_{0}-x^{*}\right\|^{2},
\end{aligned}
$$

which implies that

$$
U_{k-1} x_{0}=S_{k} U_{k-1} x_{0}
$$

for every $k=1,2, \ldots, N-1$.

From (2.2), it implies that $x_{0}=S_{1} x_{0}$, that is, $x_{0} \in F(S)$. From the definition of $S^{A}$, we have

$$
U_{1} x_{0}=T_{1}\left(\alpha_{1}^{1} S_{1} U_{0} x_{0}+\alpha_{2}^{1} U_{0} x_{0}+\alpha_{3}^{1} x_{0}\right)=T_{1} x_{0}=x_{0}
$$

From (2.2) and $U_{1} x_{0}=x_{0}$, we have

$$
\begin{aligned}
\left\|x_{0}-x^{*}\right\|^{2} \leq & \prod_{j=3}^{N}\left(1-\alpha_{3}^{j}\right)\left(( 1 - \alpha _ { 3 } ^ { 2 } ) \left(\left\|U_{1} x_{0}-x^{*}\right\|^{2}\right.\right. \\
& \left.-2 \frac{\alpha_{1}^{2}}{1-\alpha_{3}^{2}}\left(\kappa-K^{2}\left(\frac{\alpha_{1}^{2}}{1-\alpha_{3}^{2}}\right)\right)\left\|\left(I-S_{2}\right) U_{1} x_{0}\right\|^{2}\right) \\
& \left.+\alpha_{3}^{2}\left\|x_{0}-x^{*}\right\|^{2}\right)+\left(1-\prod_{j=3}^{N}\left(1-\alpha_{3}^{j}\right)\right)\left\|x_{0}-x^{*}\right\|^{2} \\
= & \prod_{j=3}^{N}\left(1-\alpha_{3}^{j}\right)\left(( 1 - \alpha _ { 3 } ^ { 2 } ) \left(\left\|x_{0}-x^{*}\right\|^{2}\right.\right. \\
& \left.-2 \frac{\alpha_{1}^{2}}{1-\alpha_{3}^{2}}\left(\kappa-K^{2}\left(\frac{\alpha_{1}^{2}}{1-\alpha_{3}^{2}}\right)\right)\left\|\left(I-S_{2}\right) x_{0}\right\|^{2}\right) \\
& \left.+\alpha_{3}^{2}\left\|x_{0}-x^{*}\right\|^{2}\right)+\left(1-\prod_{j=3}^{N}\left(1-\alpha_{3}^{j}\right)\right)\left\|x_{0}-x^{*}\right\|^{2} \\
& \left.-2 \frac{\alpha_{1}^{2}}{1-\alpha_{3}^{2}}\left(\kappa-K^{2}\left(\frac{\alpha_{1}^{2}}{1-\alpha_{3}^{2}}\right)\right)\left\|\left(I-S_{2}\right) x_{0}\right\|^{2}\right) \\
= & \prod_{j=3}^{N}\left(1-\alpha_{3}^{j}\right)\left(1-\alpha_{3}^{2}\right)\left(\left\|x_{0}-x^{*}\right\|^{2}\right. \\
&
\end{aligned}
$$




$$
\begin{aligned}
& +\prod_{j=3}^{N}\left(1-\alpha_{3}^{j}\right) \alpha_{3}^{2}\left\|x_{0}-x^{*}\right\|^{2}+\left(1-\prod_{j=3}^{N}\left(1-\alpha_{3}^{j}\right)\right)\left\|x_{0}-x^{*}\right\|^{2} \\
= & \prod_{j=2}^{N}\left(1-\alpha_{3}^{j}\right)\left(\left\|x_{0}-x^{*}\right\|^{2}-2 \frac{\alpha_{1}^{2}}{1-\alpha_{3}^{2}}\left(\kappa-K^{2}\left(\frac{\alpha_{1}^{2}}{1-\alpha_{3}^{2}}\right)\right)\left\|\left(I-S_{2}\right) x_{0}\right\|^{2}\right) \\
& +\left(1-\prod_{j=2}^{N}\left(1-\alpha_{3}^{j}\right)\right)\left\|x_{0}-x^{*}\right\|^{2} .
\end{aligned}
$$

It implies that $x_{0}=S_{2} x_{0}$.

From the definition of $S^{A}$ and $x_{0}=S_{2} x_{0}$, we have

$$
U_{2} x_{0}=T_{2}\left(\alpha_{1}^{2} S_{2} U_{1}+\alpha_{2}^{2} U_{1}+\alpha_{3}^{2} I\right) x_{0}=T_{2} x_{0} .
$$

From the definition of $U_{3}$ and (2.4), we have

$$
U_{3} x_{0}=T_{3}\left(\alpha_{1}^{3} S_{3} U_{2}+\alpha_{2}^{3} U_{2}+\alpha_{3}^{3} I\right) x_{0}=T_{3}\left(\left(1-\alpha_{3}^{3}\right) U_{2} x_{0}+\alpha_{3}^{3} x_{0}\right) .
$$

From (2.2), (2.6), (2.7) and $E$ is uniformly convex, we have

$$
\begin{aligned}
& \left\|x_{0}-x^{*}\right\|^{2} \leq \prod_{j=4}^{N}\left(1-\alpha_{3}^{j}\right)\left\|U_{3} x_{0}-x^{*}\right\|^{2}+\left(1-\prod_{j=4}^{N}\left(1-\alpha_{3}^{j}\right)\right)\left\|x_{0}-x^{*}\right\|^{2} \\
& =\prod_{j=4}^{N}\left(1-\alpha_{3}^{j}\right)\left\|T_{3}\left(\left(1-\alpha_{3}^{3}\right) U_{2} x_{0}+\alpha_{3}^{3} x_{0}\right)-x^{*}\right\|^{2} \\
& +\left(1-\prod_{j=4}^{N}\left(1-\alpha_{3}^{j}\right)\right)\left\|x_{0}-x^{*}\right\|^{2} \\
& \leq \prod_{j=4}^{N}\left(1-\alpha_{3}^{j}\right)\left\|\left(1-\alpha_{3}^{3}\right)\left(U_{2} x_{0}-x^{*}\right)+\alpha_{3}^{3}\left(x_{0}-x^{*}\right)\right\|^{2} \\
& +\left(1-\prod_{j=4}^{N}\left(1-\alpha_{3}^{j}\right)\right)\left\|x_{0}-x^{*}\right\|^{2} \\
& =\prod_{j=4}^{N}\left(1-\alpha_{3}^{j}\right)\left\|\left(1-\alpha_{3}^{3}\right)\left(T_{2} x_{0}-x^{*}\right)+\alpha_{3}^{3}\left(x_{0}-x^{*}\right)\right\|^{2} \\
& +\left(1-\prod_{j=4}^{N}\left(1-\alpha_{3}^{j}\right)\right)\left\|x_{0}-x^{*}\right\|^{2} \\
& \leq \prod_{j=4}^{N}\left(1-\alpha_{3}^{j}\right)\left(\left(1-\alpha_{3}^{3}\right)\left\|T_{2} x_{0}-x^{*}\right\|^{2}+\alpha_{3}^{3}\left\|x_{0}-x^{*}\right\|^{2}\right. \\
& \left.-\alpha_{3}^{3}\left(1-\alpha_{3}^{3}\right) g_{2}\left(\left\|T_{2} x_{0}-x_{0}\right\|\right)\right) \\
& +\left(1-\prod_{j=4}^{N}\left(1-\alpha_{3}^{j}\right)\right)\left\|x_{0}-x^{*}\right\|^{2}
\end{aligned}
$$




$$
\begin{aligned}
\leq & \prod_{j=4}^{N}\left(1-\alpha_{3}^{j}\right)\left(\left\|x_{0}-x^{*}\right\|^{2}-\alpha_{3}^{3}\left(1-\alpha_{3}^{3}\right) g_{2}\left(\left\|T_{2} x_{0}-x_{0}\right\|\right)\right) \\
& +\left(1-\prod_{j=4}^{N}\left(1-\alpha_{3}^{j}\right)\right)\left\|x_{0}-x^{*}\right\|^{2} .
\end{aligned}
$$

It implies that

$$
g_{2}\left(\left\|T_{2} x_{0}-x_{0}\right\|\right)=0
$$

Assume that $T_{2} x_{0} \neq x_{0}$, then we have $\left\|T_{2} x_{0}-x_{0}\right\|>0$. From the properties of $g_{2}$, we have

$$
0=g(0)<g\left(\left\|T_{2} x_{0}-x_{0}\right\|\right)=0 .
$$

This is a contradiction. Then we have $T_{2} x_{0}=x_{0}$. From (2.6), we have $x_{0}=T_{2} x_{0}=U_{2} x_{0}$.

From the definition of $U_{3}$, we have

$$
U_{3} x_{0}=T_{3}\left(\left(1-\alpha_{3}^{3}\right) U_{2} x_{0}+\alpha_{3}^{3} x_{0}\right)=T_{3} x_{0}
$$

By using the same method as above, we have

$$
x_{0}=U_{3} x_{0}=T_{3} x_{0}
$$

Continuing on this way, we can conclude that

$$
x_{0}=U_{i} x_{0}=T_{i} x_{0}
$$

for every $i=1,2, \ldots, N-1$. From (2.2) and (2.10), we have

$$
\begin{aligned}
\left\|x_{0}-x^{*}\right\|^{2} \leq & \left(1-\alpha_{3}^{N}\right)\left(\left\|U_{N-1} x_{0}-x^{*}\right\|^{2}\right. \\
& \left.-2 \frac{\alpha_{1}^{N}}{1-\alpha_{3}^{N}}\left(\kappa-K^{2}\left(\frac{\alpha_{1}^{N}}{1-\alpha_{3}^{N}}\right)\right)\left\|\left(I-S_{N}\right) U_{N-1} x_{0}\right\|^{2}\right)+\alpha_{3}^{N}\left\|x_{0}-x^{*}\right\|^{2} \\
= & \left(1-\alpha_{3}^{N}\right)\left(\left\|x_{0}-x^{*}\right\|^{2}-2 \frac{\alpha_{1}^{N}}{1-\alpha_{3}^{N}}\left(\kappa-K^{2}\left(\frac{\alpha_{1}^{N}}{1-\alpha_{3}^{N}}\right)\right)\left\|\left(I-S_{N}\right) x_{0}\right\|^{2}\right) \\
& +\alpha_{3}^{N}\left\|x_{0}-x^{*}\right\|^{2} .
\end{aligned}
$$

It implies that

$$
x_{0}=S_{N} x_{0} .
$$

From the definition of $S^{A}$ and (2.10), we have

$$
x_{0}=S^{A} x_{0}=U_{N} x_{0}=T_{N}\left(\alpha_{1}^{N} S_{N} U_{N-1}+\alpha_{2}^{N} U_{N-1}+\alpha_{3}^{N} I\right) x_{0}=T_{N} x_{0} .
$$


Then we have

$$
x_{0} \in \bigcap_{i=1}^{N} F\left(T_{i}\right) \quad \text { and } \quad x_{0} \in \bigcap_{i=1}^{N} F\left(U_{i}\right) \text {. }
$$

Since $S_{k} U_{k-1} x_{0}=U_{k-1} x_{0}$ for every $k=1,2, \ldots, N-1$ and $x_{0} \in \bigcap_{i=1}^{N} F\left(U_{i}\right)$, then we have

$$
S_{k} x_{0}=x_{0}
$$

for every $k=1,2, \ldots, N-1$. From (2.11), it implies that

$$
x_{0} \in \bigcap_{i=1}^{N} F\left(S_{i}\right)
$$

From (2.12) and (2.13), we have

$$
x_{0} \in \bigcap_{i=1}^{N} F\left(T_{i}\right) \cap \bigcap_{i=1}^{N} F\left(S_{i}\right) .
$$

Hence, $F\left(S^{A}\right) \subseteq \bigcap_{i=1}^{N} F\left(T_{i}\right) \cap \bigcap_{i=1}^{N} F\left(S_{i}\right)$. It is easy to see that $\bigcap_{i=1}^{N} F\left(T_{i}\right) \cap \bigcap_{i=1}^{N} F\left(S_{i}\right) \subseteq F\left(S^{A}\right)$. Applying (2.2), we have that the mapping $S^{A}$ is nonexpansive.

Lemma 2.8 [19] Let $C$ be a closed convex subset of a strictly convex Banach space E. Let $T_{1}$ and $T_{2}$ be two nonexpansive mappings from $C$ into itself with $F\left(T_{1}\right) \cap F\left(T_{2}\right) \neq \emptyset$. Define a mapping $S$ by

$$
S x=\lambda T_{1} x+(1-\lambda) T_{2} x, \quad \forall x \in C,
$$

where $\lambda$ is a constant in $(0,1)$. Then $S$ is nonexpansive and $F(S)=F\left(T_{1}\right) \cap F\left(T_{2}\right)$.

Applying Lemma 2.8, we have the following lemma.

Lemma 2.9 Let $C$ be a closed convex subset of a strictly convex Banach space E. Let $T_{1}, T_{2}$ and $T_{3}$ be three nonexpansive mappings from $C$ into itself with $F\left(T_{1}\right) \cap F\left(T_{2}\right) \cap F\left(T_{3}\right) \neq \emptyset$. Define a mapping $S$ by

$$
S x=\alpha T_{1} x+\beta T_{2} x+\gamma T_{3} x, \quad \forall x \in C,
$$

where $\alpha, \beta, \gamma$ is a constant in $(0,1)$ and $\alpha+\beta+\gamma=1$. Then $S$ is nonexpansive and $F(S)=$ $F\left(T_{1}\right) \cap F\left(T_{2}\right) \cap F\left(T_{3}\right)$.

Proof For every $x \in C$ and the definition of the mapping $S$, we have

$$
\begin{aligned}
S x & =\alpha T_{1} x+\beta T_{2} x+\gamma T_{3} x \\
& =\alpha T_{1} x+(1-\alpha)\left(\frac{\beta}{1-\alpha} T_{2} x+\frac{\gamma}{1-\alpha} T_{3} x\right)
\end{aligned}
$$




$$
\begin{aligned}
& =\alpha T_{1} x+(1-\alpha)\left(\frac{\beta}{1-\alpha} T_{2} x+\left(1-\frac{\beta}{1-\alpha}\right) T_{3} x\right) \\
& =\alpha T_{1} x+(1-\alpha) S_{1} x
\end{aligned}
$$

where $S_{1}=\frac{\beta}{1-\alpha} T_{2}+\left(1-\frac{\beta}{1-\alpha}\right) T_{3}$. From Lemma 2.8, we have $F\left(S_{1}\right)=F\left(T_{2}\right) \cap F\left(T_{3}\right)$ and $S_{1}$ is a nonexpansive mapping. From Lemma 2.8 and (2.15), we have $F(S)=F\left(T_{1}\right) \cap F\left(S_{1}\right)$ and $S$ is a nonexpansive mapping. Hence we have $F(S)=F\left(T_{1}\right) \cap F\left(T_{2}\right) \cap F\left(T_{3}\right)$.

\section{Main results}

Theorem 3.1 Let $C$ be a nonempty closed convex subset of a uniformly convex and 2-uniformly smooth Banach space $E$. Let $Q_{C}$ be a sunny nonexpansive retraction from $E$ onto $C$ and let $A, B$ be $\alpha$ - and $\beta$-inverse strongly accretive mappings of $C$ into $E$, respectively. Let $\left\{S_{i}\right\}_{i=1}^{N}$ be a finite family of $\kappa_{i}$-strict pseudo-contractions of $C$ into itself and let $\left\{T_{i}\right\}_{i=1}^{N}$ be a finite family of nonexpansive mappings of $C$ into itself with $\mathcal{F}=\bigcap_{i=1}^{N} F\left(S_{i}\right) \cap$ $\bigcap_{i=1}^{N} F\left(T_{i}\right) \cap S(C, A) \cap S(C, B) \neq \emptyset$ and $\kappa=\min \left\{\kappa_{i}: i=1,2, \ldots, N\right\}$ with $K^{2} \leq \kappa$, where $K$ is the 2-uniformly smooth constant of $E$. Let $\alpha_{j}=\left(\alpha_{1}^{j}, \alpha_{2}^{j}, \alpha_{3}^{j}\right) \in I \times I \times I$, where $I=[0,1]$, $\alpha_{1}^{j}+\alpha_{2}^{j}+\alpha_{3}^{j}=1, \alpha_{1}^{j} \in(0,1], \alpha_{2}^{j} \in[0,1]$ and $\alpha_{3}^{j} \in(0,1)$ for all $j=1,2, \ldots, N$. Let $S^{A}$ be the $S^{A}$-mapping generated by $S_{1}, S_{2}, \ldots, S_{N}, T_{1}, T_{2}, \ldots, T_{N}$ and $\alpha_{1}, \alpha_{2}, \ldots, \alpha_{N}$. Let $\left\{x_{n}\right\}$ be the sequence generated by $x_{1}, u \in C$ and

$$
x_{n+1}=\alpha_{n} u+\beta_{n} x_{n}+\gamma_{n} Q_{C}(I-a A) x_{n}+\delta_{n} Q_{C}(I-b B) x_{n}+\eta_{n} S^{A} x_{n}, \quad \forall n \geq 1,
$$

where $\left\{\alpha_{n}\right\},\left\{\beta_{n}\right\},\left\{\gamma_{n}\right\},\left\{\delta_{n}\right\},\left\{\eta_{n}\right\} \in[0,1]$ and $\alpha_{n}+\beta_{n}+\gamma_{n}+\delta_{n}+\eta_{n}=1$ and satisfy the following conditions:

(i) $\quad \lim _{n \rightarrow \infty} \alpha_{n}=0, \quad \sum_{n=1}^{\infty} \alpha_{n}=\infty$,

(ii) $\left\{\gamma_{n}\right\},\left\{\delta_{n}\right\},\left\{\eta_{n}\right\} \subseteq[c, d] \subset(0,1)$, for some $c, d>0, \forall n \geq 1$,

(iii) $\quad \sum_{n=1}^{\infty}\left|\beta_{n+1}-\beta_{n}\right|, \quad \sum_{n=1}^{\infty}\left|\gamma_{n+1}-\gamma_{n}\right|, \quad \sum_{n=1}^{\infty}\left|\delta_{n+1}-\delta_{n}\right|$, $\sum_{n=1}^{\infty}\left|\eta_{n+1}-\eta_{n}\right|, \quad \sum_{n=1}^{\infty}\left|\alpha_{n+1}-\alpha_{n}\right|<\infty$,

(iv) $0<\liminf _{n \rightarrow \infty} \beta_{n} \leq \limsup _{n \rightarrow \infty} \beta_{n}<1$,

(v) $a \in\left(0, \frac{\alpha}{K^{2}}\right)$ and $b \in\left(0, \frac{\beta}{K^{2}}\right)$.

Then $\left\{x_{n}\right\}$ converges strongly to $z_{0}=Q_{\mathcal{F}} u$, where $Q_{\mathcal{F}}$ is the sunny nonexpansive retraction of $C$ onto $\mathcal{F}$.

Proof First we show that $Q_{C}(I-a A)$ and $Q_{C}(I-b B)$ are nonexpansive mappings. Let $x, y \in C$, we have

$$
\begin{aligned}
\left\|Q_{C}(I-a A) x-Q_{C}(I-a A) y\right\|^{2} & \leq\|x-y-a(A x-A y)\|^{2} \\
& \leq\|x-y\|^{2}-2 a\langle A x-A y, j(x-y)\rangle+2 K^{2} a^{2}\|A x-A y\|^{2}
\end{aligned}
$$




$$
\begin{aligned}
& \leq\|x-y\|^{2}-2 a \alpha\|A x-A y\|^{2}+2 K^{2} a^{2}\|A x-A y\|^{2} \\
& =\|x-y\|^{2}-2 a\left(\alpha-K^{2} a\right)\|A x-A y\|^{2} \\
& \leq\|x-y\|^{2} .
\end{aligned}
$$

Then we have $Q_{C}(I-a A)$ is a nonexpansive mapping. By using the same methods as (3.2), we have $Q_{C}(I-b B)$ is a nonexpansive mapping.

Let $x^{*} \in \mathcal{F}$. From Lemma 2.3, we have $x^{*} \in F\left(Q_{C}(I-a A)\right)$ and $x^{*} \in F\left(Q_{C}(I-b B)\right)$. By the definition of $x_{n}$, we have

$$
\begin{aligned}
\left\|x_{n+1}-x^{*}\right\| \leq & \alpha_{n}\left\|u-x^{*}\right\|+\beta_{n}\left\|x_{n}-x^{*}\right\|+\gamma_{n}\left\|Q_{C}(I-a A) x_{n}-x^{*}\right\| \\
& +\delta_{n}\left\|Q_{C}(I-b B) x_{n}-x^{*}\right\|+\eta_{n}\left\|S^{A} x_{n}-x^{*}\right\| \\
\leq & \alpha_{n}\left\|u-x^{*}\right\|+\left(1-\alpha_{n}\right)\left\|x_{n}-x^{*}\right\| \\
\leq & \max \left\{\left\|u-x^{*}\right\|,\left\|x_{1}-x^{*}\right\|\right\} .
\end{aligned}
$$

By induction, we have $\left\|x_{n}-x^{*}\right\| \leq \max \left\{\left\|u-x^{*}\right\|,\left\|x_{1}-x^{*}\right\|\right\}$. We can imply that the sequence $\left\{x_{n}\right\}$ is bounded and so are $\left\{S^{A} x_{n}\right\},\left\{Q_{C}(I-a A) x_{n}\right\}$ and $\left\{Q_{C}(I-b B) x_{n}\right\}$.

Next, we show that $\lim _{n \rightarrow \infty}\left\|x_{n+1}-x_{n}\right\|=0$. From the definition of $x_{n}$, we have

$$
\begin{aligned}
\left\|x_{n+1}-x_{n}\right\|= & \| \alpha_{n} u+\beta_{n} x_{n}+\gamma_{n} Q_{C}(I-a A) x_{n}+\delta_{n} Q_{C}(I-b B) x_{n}+\eta_{n} S^{A} x_{n} \\
& -\alpha_{n-1} u-\beta_{n-1} x_{n-1}-\gamma_{n-1} Q_{C}(I-a A) x_{n-1}-\delta_{n-1} Q_{C}(I-b B) x_{n-1} \\
& -\eta_{n-1} S^{A} x_{n-1} \| \\
\leq & \left|\alpha_{n}-\alpha_{n-1}\right|\|u\|+\beta_{n}\left\|x_{n}-x_{n-1}\right\|+\left|\beta_{n}-\beta_{n-1}\right|\left\|x_{n-1}\right\| \\
& +\gamma_{n}\left\|Q_{C}(I-a A) x_{n}-Q_{C}(I-a A) x_{n-1}\right\|+\left|\gamma_{n}-\gamma_{n-1}\right|\left\|Q_{C}(I-a A) x_{n-1}\right\| \\
& +\delta_{n}\left\|Q_{C}(I-b B) x_{n}-Q_{C}(I-b B) x_{n-1}\right\|+\left|\delta_{n}-\delta_{n-1}\right|\left\|Q_{C}(I-b B) x_{n-1}\right\| \\
& +\eta_{n}\left\|S^{A} x_{n}-S^{A} x_{n-1}\right\|+\left|\eta_{n-1}-\eta_{n}\right|\left\|S^{A} x_{n}\right\| \\
\leq & \left(1-\alpha_{n}\right)\left\|x_{n}-x_{n-1}\right\|+\left|\alpha_{n}-\alpha_{n-1}\right|\|u\|+\left|\beta_{n}-\beta_{n-1}\right|\left\|x_{n-1}\right\| \\
& +\left|\gamma_{n}-\gamma_{n-1}\right|\left\|Q_{C}(I-a A) x_{n-1}\right\|+\left|\delta_{n}-\delta_{n-1}\right|\left\|Q_{C}(I-b B) x_{n-1}\right\| \\
& +\left|\eta_{n-1}-\eta_{n}\right|\left\|S^{A} x_{n}\right\| .
\end{aligned}
$$

Applying Lemma 2.6, we have

$$
\lim _{n \rightarrow \infty}\left\|x_{n+1}-x_{n}\right\|=0 .
$$

Next, we show that

$$
\lim _{n \rightarrow \infty}\left\|Q_{C}(I-a A) x_{n}-x_{n}\right\|=\lim _{n \rightarrow \infty}\left\|Q_{C}(I-b B) x_{n}-x_{n}\right\|=\lim _{n \rightarrow \infty}\left\|S^{A} x_{n}-x_{n}\right\|=0 .
$$

From the definition of $x_{n}$, we have

$$
\begin{aligned}
\left\|x_{n+1}-x^{*}\right\|^{2}= & \| \alpha_{n}\left(u-x^{*}\right)+\beta_{n}\left(x_{n}-x^{*}\right)+\gamma_{n}\left(Q_{C}(I-a A) x_{n}-x^{*}\right) \\
& +\delta_{n}\left(Q_{C}(I-b B) x_{n}-x^{*}\right)+\eta_{n}\left(S^{A} x_{n}-x^{*}\right) \|^{2}
\end{aligned}
$$




$$
\begin{aligned}
= & \| \beta_{n}\left(x_{n}-x^{*}\right)+\gamma_{n}\left(Q_{C}(I-a A) x_{n}-x^{*}\right)+\left(\alpha_{n}+\delta_{n}+\eta_{n}\right)\left(\frac{\alpha_{n}\left(u-x^{*}\right)}{\alpha_{n}+\delta_{n}+\eta_{n}}\right. \\
& \left.+\frac{\delta_{n}\left(Q_{C}(I-b B) x_{n}-x^{*}\right)}{\alpha_{n}+\delta_{n}+\eta_{n}}+\frac{\eta_{n}\left(S^{A} x_{n}-x^{*}\right)}{\alpha_{n}+\delta_{n}+\eta_{n}}\right) \|^{2} \\
= & \left\|\beta_{n}\left(x_{n}-x^{*}\right)+\gamma_{n}\left(Q_{C}(I-a A) x_{n}-x^{*}\right)+c_{n} z_{n}\right\|^{2},
\end{aligned}
$$

where $c_{n}=\alpha_{n}+\delta_{n}+\eta_{n}$ and $z_{n}=\frac{\alpha_{n}\left(u-x^{*}\right)}{\alpha_{n}+\delta_{n}+\eta_{n}}+\frac{\delta_{n}\left(Q_{C}(I-b B) x_{n}-x^{*}\right)}{\alpha_{n}+\delta_{n}+\eta_{n}}+\frac{\eta_{n}\left(S^{A} x_{n}-x^{*}\right)}{\alpha_{n}+\delta_{n}+\eta_{n}}$.

From Lemma 2.2, we have

$$
\begin{aligned}
\left\|x_{n+1}-x^{*}\right\|^{2} \leq & \beta_{n}\left\|x_{n}-x^{*}\right\|^{2}+\gamma_{n}\left\|Q_{C}(I-a A) x_{n}-x^{*}\right\|+c_{n}\left\|z_{n}\right\|^{2} \\
& -\beta_{n} \gamma_{n} g_{1}\left(\left\|x_{n}-Q_{C}(I-a A) x_{n}\right\|\right) \\
\leq & \left(\beta_{n}+\gamma_{n}\right)\left\|x_{n}-x^{*}\right\|^{2}-\beta_{n} \gamma_{n} g_{1}\left(\left\|x_{n}-Q_{C}(I-a A) x_{n}\right\|\right) \\
& +c_{n}\left(\frac{\alpha_{n}\left\|u-x^{*}\right\|^{2}}{\alpha_{n}+\delta_{n}+\eta_{n}}+\frac{\delta_{n}\left\|Q_{C}(I-b B) x_{n}-x^{*}\right\|^{2}}{\alpha_{n}+\delta_{n}+\eta_{n}}+\frac{\eta_{n}\left\|S^{A} x_{n}-x^{*}\right\|^{2}}{\alpha_{n}+\delta_{n}+\eta_{n}}\right) \\
\leq & \left(\beta_{n}+\gamma_{n}\right)\left\|x_{n}-x^{*}\right\|^{2}-\beta_{n} \gamma_{n} g_{1}\left(\left\|x_{n}-Q_{C}(I-a A) x_{n}\right\|\right) \\
& +\alpha_{n}\left\|u-x^{*}\right\|^{2}+\left(\delta_{n}+\eta_{n}\right)\left\|x_{n}-x^{*}\right\|^{2} \\
\leq & \left\|x_{n}-x^{*}\right\|^{2}-\beta_{n} \gamma_{n} g_{1}\left(\left\|x_{n}-Q_{C}(I-a A) x_{n}\right\|\right)+\alpha_{n}\left\|u-x^{*}\right\|^{2},
\end{aligned}
$$

which implies that

$$
\begin{aligned}
\beta_{n} \gamma_{n} g_{1}\left(\left\|x_{n}-Q_{C}(I-a A) x_{n}\right\|\right) \leq & \left\|x_{n}-x^{*}\right\|^{2}-\left\|x_{n+1}-x^{*}\right\|^{2}+\alpha_{n}\left\|u-x^{*}\right\|^{2} \\
\leq & \left(\left\|x_{n}-x^{*}\right\|+\left\|x_{n+1}-x^{*}\right\|\right)\left\|x_{n+1}-x_{n}\right\| \\
& +\alpha_{n}\left\|u-x^{*}\right\|^{2} .
\end{aligned}
$$

From (3.3) and condition (i), we obtain

$$
\lim _{n \rightarrow \infty} g_{1}\left(\left\|x_{n}-Q_{C}(I-a A) x_{n}\right\|\right)=0 .
$$

From the property of $g_{1}$, we have

$$
\lim _{n \rightarrow \infty}\left\|x_{n}-Q_{C}(I-a A) x_{n}\right\|=0
$$

By using the same method as (3.7), we can imply that

$$
\lim _{n \rightarrow \infty}\left\|x_{n}-Q_{C}(I-b B) x_{n}\right\|=\lim _{n \rightarrow \infty}\left\|x_{n}-S^{A} x_{n}\right\|=0 .
$$

Define $G x=\alpha S^{A} x+\beta Q_{C}(I-a A) x+\gamma Q_{C}(I-b B) x$ for all $x \in C$ and $\alpha+\beta+\gamma=1$. From Lemma 2.9, we have $F(G)=F\left(Q_{C}(I-a A)\right) \cap F\left(Q_{C}(I-b B)\right) \cap F\left(S^{A}\right)$. From Lemmas 2.3 and 2.7, we have $\mathcal{F}=F(G)=\bigcap_{i=1}^{N} F\left(T_{i}\right) \cap \bigcap_{i=1}^{N} F\left(S_{i}\right) \cap S(C, A) \cap S(C, B)$. By the definition of $G$, we obtain

$$
\left\|G x_{n}-x_{n}\right\| \leq \alpha\left\|S^{A} x_{n}-x_{n}\right\|+\beta\left\|Q_{C}(I-a A) x_{n}-x_{n}\right\|+\gamma\left\|Q_{C}(I-b B) x_{n}-x_{n}\right\| .
$$


From (3.4), we have

$$
\lim _{n \rightarrow \infty}\left\|G x_{n}-x_{n}\right\|=0 .
$$

From Lemma 2.5 and (3.8), we have

$$
\limsup _{n \rightarrow \infty}\left\langle u-z_{0}, j\left(x_{n}-z_{0}\right)\right\rangle \leq 0,
$$

where $z_{0}=Q_{\mathcal{F}} u$. Finally, we prove strong convergence of the sequence $\left\{x_{n}\right\}$ to $z_{0}=Q_{\mathcal{F}} u$. From the definition of $x_{n}$, we have

$$
\begin{aligned}
\left\|x_{n+1}-z_{0}\right\|^{2}= & \| \alpha_{n}\left(u-z_{0}\right)+\beta_{n}\left(x_{n}-z_{0}\right)+\gamma_{n}\left(Q_{C}(I-a A) x_{n}-z_{0}\right) \\
& +\delta_{n}\left(Q_{C}(I-b B) x_{n}-z_{0}\right)+\eta_{n}\left(S^{A} x_{n}-z_{0}\right) \|^{2} \\
= & \| \alpha_{n}\left(u-z_{0}\right)+\left(1-\alpha_{n}\right)\left(\frac{\beta_{n}\left(x_{n}-z_{0}\right)}{1-\alpha_{n}}+\frac{\gamma_{n}\left(Q_{C}(I-a A) x_{n}-z_{0}\right)}{1-\alpha_{n}}\right. \\
& \left.+\frac{\delta_{n}\left(Q_{C}(I-b B) x_{n}-z_{0}\right)}{1-\alpha_{n}}+\frac{\eta_{n}\left(S^{A} x_{n}-z_{0}\right)}{1-\alpha_{n}}\right) \|^{2} \\
\leq & \|\left(1-\alpha_{n}\right)\left(\frac{\beta_{n}\left(x_{n}-z_{0}\right)}{1-\alpha_{n}}+\frac{\gamma_{n}\left(Q_{C}(I-a A) x_{n}-z_{0}\right)}{1-\alpha_{n}}\right. \\
& \left.+\frac{\delta_{n}\left(Q_{C}(I-b B) x_{n}-z_{0}\right)}{1-\alpha_{n}}+\frac{\eta_{n}\left(S^{A} x_{n}-z_{0}\right)}{1-\alpha_{n}}\right) \|^{2}+2 \alpha_{n}\left\langle u-x_{0}, j\left(x_{n+1}-z_{0}\right)\right\rangle \\
\leq & \left(1-\alpha_{n}\right)\left\|x_{n}-z_{0}\right\|^{2}+2 \alpha_{n}\left\langle u-x_{0}, j\left(x_{n+1}-z_{0}\right)\right\rangle .
\end{aligned}
$$

Applying Lemma 2.6 and condition (i), we have $\lim _{n \rightarrow \infty}\left\|x_{n}-z_{0}\right\|=0$. This completes the proof.

\section{Applications}

From our main results, we obtain strong convergence theorems in a Banach space. Before proving these theorem, we need the following lemma which is the result from Lemma 2.7 and Definition 1.4. Therefore, we omit the proof.

Lemma 4.1 Let $C$ be a nonempty closed convex subset of a 2-uniformly smooth and uniformly convex Banach space. Let $\left\{S_{i}\right\}_{i=1}^{N}$ be a finite family of $\kappa_{i}$-strict pseudo-contractions of $C$ into itself with $\bigcap_{i=1}^{N} F\left(S_{i}\right) \neq \emptyset$ and $\kappa=\min \left\{\kappa_{i}: i=1,2, \ldots, N\right\}$ with $K^{2} \leq \kappa$, where $K$ is the 2-uniformly smooth constant of E. Let $\alpha_{j}=\left(\alpha_{1}^{j}, \alpha_{2}^{j}, \alpha_{3}^{j}\right) \in I \times I \times I$, where $I=[0,1]$, $\alpha_{1}^{j}+\alpha_{2}^{j}+\alpha_{3}^{j}=1, \alpha_{1}^{j} \in(0,1], \alpha_{2}^{j} \in[0,1]$ and $\alpha_{3}^{j} \in(0,1)$ for all $j=1,2, \ldots, N$. Let $S$ be the $S-$ mapping generated by $S_{1}, S_{2}, \ldots, S_{N}$ and $\alpha_{1}, \alpha_{2}, \ldots, \alpha_{N}$. Then $F(S)=\bigcap_{i=1}^{N} F\left(S_{i}\right)$ and $S$ is a nonexpansive mapping.

Theorem 4.2 Let $C$ be a nonempty closed convex subset of a uniformly convex and 2uniformly smooth Banach space $E$. Let $Q_{C}$ be a sunny nonexpansive retraction from $E$ onto $C$ and let $A, B$ be $\alpha$-and $\beta$-inverse strongly accretive mappings of $C$ into $E$, respectively. Let $\left\{S_{i}\right\}_{i=1}^{N}$ be a finite family of $\kappa_{i}$-strict pseudo-contractions of $C$ into itself with $\mathcal{F}=\bigcap_{i=1}^{N} F\left(S_{i}\right) \cap S(C, A) \cap S(C, B) \neq \emptyset$ and $\kappa=\min \left\{\kappa_{i}: i=1,2, \ldots, N\right\}$ with $K^{2} \leq \kappa$, where $K$ is the 2-uniformly smooth constant of E. Let $\alpha_{j}=\left(\alpha_{1}^{j}, \alpha_{2}^{j}, \alpha_{3}^{j}\right) \in I \times I \times I$, where $I=[0,1]$, 
$\alpha_{1}^{j}+\alpha_{2}^{j}+\alpha_{3}^{j}=1, \alpha_{1}^{j} \in(0,1], \alpha_{2}^{j} \in[0,1]$ and $\alpha_{3}^{j} \in(0,1)$ for all $j=1,2, \ldots, N$. Let $S$ be the $S$ mapping generated by $S_{1}, S_{2}, \ldots, S_{N}$ and $\alpha_{1}, \alpha_{2}, \ldots, \alpha_{N}$. Let $\left\{x_{n}\right\}$ be the sequence generated by $x_{1}, u \in C$ and

$$
x_{n+1}=\alpha_{n} u+\beta_{n} x_{n}+\gamma_{n} Q_{C}(I-a A) x_{n}+\delta_{n} Q_{C}(I-b B) x_{n}+\eta_{n} S x_{n}, \quad \forall n \geq 1,
$$

where $\left\{\alpha_{n}\right\},\left\{\beta_{n}\right\},\left\{\gamma_{n}\right\},\left\{\delta_{n}\right\},\left\{\eta_{n}\right\} \in[0,1]$ and $\alpha_{n}+\beta_{n}+\gamma_{n}+\delta_{n}+\eta_{n}=1$ and satisfy the following conditions:

(i) $\quad \lim _{n \rightarrow \infty} \alpha_{n}=0, \quad \sum_{n=1}^{\infty} \alpha_{n}=\infty$,

(ii) $\left\{\gamma_{n}\right\},\left\{\delta_{n}\right\},\left\{\eta_{n}\right\} \subseteq[c, d] \subset(0,1)$ for some $c, d>0, \forall n \geq 1$,

(iii) $\quad \sum_{n=1}^{\infty}\left|\beta_{n+1}-\beta_{n}\right|, \quad \sum_{n=1}^{\infty}\left|\gamma_{n+1}-\gamma_{n}\right|, \quad \sum_{n=1}^{\infty}\left|\delta_{n+1}-\delta_{n}\right|$,

$\sum_{n=1}^{\infty}\left|\eta_{n+1}-\eta_{n}\right|, \quad \sum_{n=1}^{\infty}\left|\alpha_{n+1}-\alpha_{n}\right|<\infty$,

(iv) $\quad 0<\liminf _{n \rightarrow \infty} \beta_{n} \leq \limsup _{n \rightarrow \infty} \beta_{n}<1$,

(v) $a \in\left(0, \frac{\alpha}{K^{2}}\right)$ and $b \in\left(0, \frac{\beta}{K^{2}}\right)$.

Then $\left\{x_{n}\right\}$ converges strongly to $z_{0}=Q_{\mathcal{F}} u$, where $Q_{\mathcal{F}}$ is the sunny nonexpansive retraction of $C$ onto $\mathcal{F}$.

Proof Put $I=T_{1}=T_{2}=\cdots=T_{N}$ in Theorem 3.1. From Lemma 4.1 and Theorem 3.1 we can conclude the desired result.

Theorem 4.3 Let $C$ be a nonempty closed convex subset of a uniformly convex and 2-uniformly smooth Banach space $E$. Let $Q_{C}$ be a sunny nonexpansive retraction from $E$ onto $C$. For every $i=1,2, \ldots, N$, let $A_{i}, A, B$ be $\alpha_{i^{-}}, \alpha$-and $\beta$-inverse strongly accretive mappings of $C$ into $E$, respectively. Define a mapping $G_{i}: C \rightarrow C$ by $Q_{C}\left(I-\lambda_{i} A_{i}\right) x=G_{i} x$, where $\lambda_{i} \in\left(0, \frac{\alpha_{i}}{K^{2}}\right), K$ is the 2-uniformly smooth constant of $E$, for all $x \in C$ and $i=1,2, \ldots, N$. Let $\left\{S_{i}\right\}_{i=1}^{N}$ be a finite family of $\kappa_{i}$-strict pseudo-contractions of $C$ into itself and with $\mathcal{F}=\bigcap_{i=1}^{N} F\left(S_{i}\right) \cap \bigcap_{i=1}^{N} S\left(C, A_{i}\right) \cap S(C, A) \cap S(C, B) \neq \emptyset$ and $\kappa=\min \left\{\kappa_{i}: i=1,2, \ldots, N\right\}$ with $K^{2} \leq \kappa$. Let $\alpha_{j}=\left(\alpha_{1}^{j}, \alpha_{2}^{j}, \alpha_{3}^{j}\right) \in I \times I \times I$, where $I=[0,1], \alpha_{1}^{j}+\alpha_{2}^{j}+\alpha_{3}^{j}=1, \alpha_{1}^{j} \in(0,1], \alpha_{2}^{j} \in[0,1]$ and $\alpha_{3}^{j} \in(0,1)$ for all $j=1,2, \ldots, N$. Let $S^{A}$ be the $S^{A}$-mapping generated by $S_{1}, S_{2}, \ldots, S_{N}$, $G_{1}, G_{2}, \ldots, G_{N}$ and $\alpha_{1}, \alpha_{2}, \ldots, \alpha_{N}$. Let $\left\{x_{n}\right\}$ be the sequence generated by $x_{1}, u \in C$ and

$$
x_{n+1}=\alpha_{n} u+\beta_{n} x_{n}+\gamma_{n} Q_{C}(I-a A) x_{n}+\delta_{n} Q_{C}(I-b B) x_{n}+\eta_{n} S^{A} x_{n}, \quad \forall n \geq 1,
$$

where $\left\{\alpha_{n}\right\},\left\{\beta_{n}\right\},\left\{\gamma_{n}\right\},\left\{\delta_{n}\right\},\left\{\eta_{n}\right\} \in[0,1]$ and $\alpha_{n}+\beta_{n}+\gamma_{n}+\delta_{n}+\eta_{n}=1$ and satisfy the following conditions:

$$
\text { (i) } \quad \lim _{n \rightarrow \infty} \alpha_{n}=0, \quad \sum_{n=1}^{\infty} \alpha_{n}=\infty
$$


(ii) $\left\{\gamma_{n}\right\},\left\{\delta_{n}\right\},\left\{\eta_{n}\right\} \subseteq[c, d] \subset(0,1)$ for some $c, d>0, \forall n \geq 1$,

(iii) $\quad \sum_{n=1}^{\infty}\left|\beta_{n+1}-\beta_{n}\right|, \quad \sum_{n=1}^{\infty}\left|\gamma_{n+1}-\gamma_{n}\right|, \quad \sum_{n=1}^{\infty}\left|\delta_{n+1}-\delta_{n}\right|$,

$$
\sum_{n=1}^{\infty}\left|\eta_{n+1}-\eta_{n}\right|, \quad \sum_{n=1}^{\infty}\left|\alpha_{n+1}-\alpha_{n}\right|<\infty,
$$

(iv) $0<\liminf _{n \rightarrow \infty} \beta_{n} \leq \limsup _{n \rightarrow \infty} \beta_{n}<1$,

(v) $a \in\left(0, \frac{\alpha}{K^{2}}\right)$ and $b \in\left(0, \frac{\beta}{K^{2}}\right)$.

Then $\left\{x_{n}\right\}$ converges strongly to $z_{0}=Q_{\mathcal{F}} u$, where $Q_{\mathcal{F}}$ is the sunny nonexpansive retraction of $C$ onto $\mathcal{F}$.

Proof By using the same method as (3.2), we can conclude that $\left\{G_{i}\right\}_{i=1}^{N}$ is a nonexpansive mapping. From Lemma 2.3, we have $F\left(G_{i}\right)=S\left(C, A_{i}\right)$ for all $i=1,2, \ldots, N$. From Theorem 3.1 we can conclude the desired conclusion.

\section{Competing interests}

The author declares that they have no competing interests.

\section{Acknowledgements}

This research was supported by the Research Administration Division of King Mongkut's Institute of Technology Ladkrabang.

\section{Received: 26 January 2013 Accepted: 28 May 2013 Published: 18 June 2013}

\section{References}

1. Sahua, DR, Petru'lel, A: Strong convergence of iterative methods by strictly pseudocontractive mappings in Banach spaces. Nonlinear Anal. 74, 6012-6023 (2011)

2. Reich, S: Asymptotic behavior of contractions in Banach spaces. J. Math. Anal. Appl. 44, 57-70 (1973)

3. Aoyama, K, liduka, H, Takahashi, W: Weak convergence of an iterative sequence for accretive operators in Banach spaces. Fixed Point Theory Appl. 2006, Article ID 35390 (2006). doi:10.1155/FPTA/2006/35390

4. Chang, SS, Joseph Lee, HW, Chan, CK: A new method for solving equilibrium problem fixed point problem and variational inequality problem with application to optimization. Nonlinear Anal. 70, 3307-3319 (2009)

5. Cai, G, Bu, S: Strong convergence theorems for variational inequality problems and fixed point problems in uniformly smooth and uniformly convex Banach spaces. J. Glob. Optim. (2012). doi:10.1007/s10898-012-9923-2

6. Jaillet, P, Lamberton, D, Lapeyre, B: Variational inequalities and the pricing of American options. Acta Appl. Math. 21 263-289 (1990)

7. Mann, WR: Mean value methods in iteration. Proc. Am. Math. Soc. 4(3), 506-510 (1953)

8. Buong, N, Lang, ND: Hybrid Mann-Halpern iteration methods for nonexpansive mappings and semigroups. Appl. Math. Comput. 218, 2459-2466 (2011)

9. Song, Y: A new sufficient condition for strong convergence of Halpern type iterations. Appl. Math. Comput. 198(2), $721-728(2007)$

10. Qin, $X$, Cho, YJ, Kang, SM, Zhou, H: Convergence of a modified Halpern-type iteration algorithm for quasi- $\boldsymbol{\phi}$-nonexpansive mappings. Appl. Math. Lett. 22, 1051-1055 (2009)

11. Zhou, $\mathrm{H}$ : Convergence theorems for $\lambda$-strict pseudo-contractions in 2-uniformly smooth Banach spaces. Nonlinear Anal. 69, 3160-3173 (2008)

12. Kangtunyakarn, A, Suantai, S: Hybrid iterative scheme for generalized equilibrium problems and fixed point problems of finite family of nonexpansive mappings. Nonlinear Anal. Hybrid Syst. 3, 296-309 (2009)

13. Kangtunyakarn, A, Suantai, S: A new mapping for finding common solutions of equilibrium problems and fixed point problems of finite family of nonexpansive mappings. Nonlinear Anal. 71, 4448-4460 (2009)

14. Kangtunyakarn, A: Iterative scheme for a nonexpansive mapping, an $\eta$-strictly pseudo-contractive mapping and variational inequality problems in a uniformly convex and 2-uniformly smooth Banach space. Fixed Point Theory Appl. 2013, Article ID 23 (2013)

15. Xu, HK: Inequalities in Banach spaces with applications. Nonlinear Anal. 16, 1127-1138 (1991)

16. Cho, YJ, Zhou, HY, Guo, G: Weak and strong convergence theorems for three-step iterations with errors for asymptotically nonexpansive mappings. Comput. Math. Appl. 47, 707-717 (2004)

17. Zhou, $\mathrm{H}$ : Convergence theorems for $\boldsymbol{\lambda}$-strict pseudocontractions in 2-uniformly smooth Banach spaces. Nonlinear Anal. 69, 3160-3173 (2008) 
18. Xu, HK: An iterative approach to quadratic optimization. J. Optim. Theory Appl. 116, 659-678 (2003)

19. Bruck, RE: Properties of fixed point sets of nonexpansive mappings in Banach spaces. Trans. Am. Math. Soc. 179 251-262 (1973)

doi:10.1186/1687-1812-2013-157

Cite this article as: Kangtunyakarn: A new mapping for finding a common element of the sets of fixed points of two finite families of nonexpansive and strictly pseudo-contractive mappings and two sets of variational inequalities in uniformly convex and 2-smooth Banach spaces. Fixed Point Theory and Applications 2013 2013:157.

Submit your manuscript to a SpringerOpen ${ }^{\odot}$ journal and benefit from:

- Convenient online submission

Rigorous peer review

Immediate publication on acceptance

- Open access: articles freely available online

- High visibility within the field

- Retaining the copyright to your article 